\title{
Oligopolistic Competition in Price and Quality
}

\author{
Andrei Dubovik' \\ Maarten Janssen ${ }^{1,2}$
}

${ }^{\prime}$ Erasmus School of Economics, Erasmus University Rotterdam, and Tinbergen Institute;

2 University of Vienna. 


\section{Tinbergen Institute}

The Tinbergen Institute is the institute for economic research of the Erasmus Universiteit Rotterdam, Universiteit van Amsterdam, and Vrije Universiteit Amsterdam.

Tinbergen Institute Amsterdam

Roetersstraat 31

1018 WB Amsterdam

The Netherlands

Tel.: +31(0)205513500

Fax: $+31(0) 205513555$

Tinbergen Institute Rotterdam

Burg. Oudlaan 50

3062 PA Rotterdam

The Netherlands

Tel.: + $31(0) 104088900$

Fax: $+31(0) 104089031$

Most TI discussion papers can be downloaded at http://www.tinbergen.nl. 


\title{
Oligopolistic competition in price and quality
}

\author{
Andrei Dubovik* \\ Maarten Janssen** \\ Tinbergen Institute \\ University Of Vienna
}

July 11, 2008

\begin{abstract}
We consider an oligopolistic market where firms compete in price and quality and where consumers are heterogeneous in knowledge: some consumers know both the prices and quality of the products offered, some know only the prices and some know neither. We show that two types of signalling equilibria are possible. Both are characterised by dispersion and Pareto-inefficiency of the price/quality offers. But, better price/quality combinations are signalled with lower prices in one type and with higher prices in the other type.
\end{abstract}

Key Words: oligopoly, competition, price, quality, imperfect information, signalling.

JEL Classification: D43, D83, L13, L15.

\footnotetext{
${ }^{*}$ Tinbergen Institute, Erasmus University Rotterdam, address: P.O. Box 1738, 3000 DR Rotterdam, The Netherlands. Webpage: http://www.yellowsite.ru/en

${ }^{* *}$ University of Vienna and Tinbergen Institute Rotterdam, address: Hohenstaufengasse 9, 1010 Vienna, Austria. Email: maarten.janssen@univie.ac.at
} 


\section{Introduction}

If there is competition between firms, then by the very nature of competition the firms will compete in as many ways as possible, and not just in price. Firms will choose such a combination of their strategic variables that it serves their interests best. However, if consumers have similar preferences and if they are fully informed about all relevant product characteristics, then all these ways of competition may be expressed in terms of a one dimensional competition model, essentially identical in nature to that of price competition.

When consumer preferences differ, however, or when some consumers are better informed than others, the competitive process involving many dimensions does not have a single dimension analogue and should be analysed in its own right. There are different approaches known in the literature dealing with price/quality competition. ${ }^{1}$ The literature where firms make strategic choices on quality can be divided into a literature dealing with consumers that differ in the amount of information they possess (see, e.g., Chan and Leland (1982), Cooper and Ross (1984) and Schwartz and Wilde (1985)) and a literature where consumers also have different preferences (see, e.g., Wolinksy (1983), Rogerson (1988) and Besancenot and Vranceanu (2004)). Another dimension along which these models differ is the type of market interaction considered. The above models either consider perfect competition or monopolistic competition.

We address the issue of price/quality competition in a strategic oligopoly model where quality is endogenously chosen and ask the following questions. First, do firms differentiate themselves with different prices and/or quality or do they make the same choices? Everyday experience suggests that we sometimes face price and/or quality dispersion. How to explain price dispersion was first addressed by Stigler (1961). The importance of quality dispersion was also emphasised, see e.g., Chan and Leland (1982). Second, can price act as a signal of quality to consumers who somehow cannot evaluate it. From previous literature with exogenous quality we know that the adverse selection problem can be mitigated if firms can signal quality choices to the consumers on the basis of the prices they charge (see, e.g., Bagwell and Riordan (1991)). Third, how should we characterise the outcomes in terms of Pareto-efficiency? As in answering these questions we want to concentrate on the role of consumers having heterogeneous information, we take their preferences to be identical.

We now discuss how we model information heterogeneity. Stigler (1961) has pointed out that acquiring information about market prices is costly.

\footnotetext{
${ }^{1}$ Here we focus only on the literature where quality is a strategic variable chosen by firms. Models with exogenous quality differences include Hertzendorf and Overgaard (2001), Daughety and Reinganum (2004), Janssen and Roy (2007). This literature mainly deals with the question whether price can signal quality in an oligopoly context.
} 
As consumers can have different search costs, different groups of consumers can be present in a market: those who know all prices and those who do not. This idea is central in Varian (1980). ${ }^{2}$ The idea also readily extends to quality, e.g. in Cooper and Ross (1984) all consumers know prices, but some of them are informed about quality while the rest is uninformed about quality. We combine these approaches in the following way. As quality is a more complex notion than price and so it is more costly for a consumer to learn the right quality than to know the price a firm charges, there are three groups of consumers in our model: those fully informed - they know the prices and quality of the products in a market, those partially informed - they know the prices but not the quality and those fully uninformed. We emphasise the role of partially informed consumers. When they are present in a market, price is not just an instrument of competition between firms, but potentially also a signalling instrument. We analyse the consequences of this informational scenario in a model where two firms choose price and quality simultaneously and consumers buy one good at most. Alternatively, firms choose quality first and then price, but are unaware of the quality choice of their competitor before they have to make their price choices. It is easy to see that this is formally equivalent to a simultaneous choice model.

We show that in this setting there can be an equilibrium characterised by a dispersion of prices and quality such that price signals quality precisely. This kind of correspondence between price and quality can be formally described as a curve in a price-quality space. Consequently, the dispersion we find we formally describe as a one-dimensional distribution of price/quality offers over that curve. In such an equilibrium partially informed consumers learn the true quality from the prices and so they act like fully informed consumers. Second, we show that the equilibrium is always such that consumers' preferences over the resulting price/quality offers are monotone in price: a consumer always prefers either the cheapest offer or the most expensive one. Which particular equilibrium is to occur depends on how marginal utility of quality changes with respect to price. If the marginal utility of quality decreases when the price gets higher, arguably a common scenario, then a consumer prefers the cheapest offer. Though the preferences over equilibrium price/quality offers are monotone in price, equilibrium quality need not be so, e.g. the quality may be worse for the average prices and better for the small and high prices. Third, we show that price/quality combinations offered in an equilibrium are Pareto-inefficient.

The paper is organised in the following way. Section 2 formally introduces the model. Section 3 provides the equilibrium analysis. Section 4 gives an example that should illustrate the more complicated expressions of

\footnotetext{
${ }^{2}$ With this idea Varian partially follows Salop and Stiglitz (1977) who get informed and uninformed consumers as a result of their model. Our model is closer to Varian in this respect because both Varian's and our model assume the presence of informed and uninformed consumers as exogenously given.
} 
section 3. Section 5 concludes. The more technical proofs of propositions are given in the appendix. In the text, we give less technical, more intuitive explanations as to why the propositions hold.

\section{Model Setup}

We consider a market with two firms selling similar products. The firms can decide both the price and quality of the product they offer. There is a unit mass of consumers who choose between the firms so as to maximise their expected utility given the information they have. The timing is as follows. First, firms simultaneously decide on the price and quality of their products. Second, each consumer decides from which firm to buy and whether to buy the product at all. We will discuss the behaviour of the firms and consumers in detail below.

\subsection{Firms}

The production technology is such that producing higher quality comes at a higher cost. For simplicity we assume a linear dependency ${ }^{3}$ so that the per-unit profits are given by

$$
\Pi(p, q)=p-a q,
$$

where $p$ and $q$ stand for price and quality and the coefficient $a>0$ characterises the quality production technology. We take $a$ to be the same across firms. For simplicity we will also assume that the firms make their production costs at the moment of sale, so there are no excess goods that are produced but not sold.

Since the firms move simultaneously a strategy of either firm is simply a distribution over all possible $(p, q)$ bundles. To describe it let $P_{i}$ and $Q_{i}$ be the random variables that stand for the price and quality offered by firm $i$ and let $\mathbb{P}_{i}$ be the probability measure that corresponds to the strategy of firm $i$. The chances that firm $i$ plays, for example, a certain $(p, q)$ bundle are then given by $\mathbb{P}\left(P_{i}=p, Q_{i}=q\right)$.

\subsection{Consumers}

We consider consumers that are homogeneous in their preferences with the preferences being represented by a utility function $U(p, q)$ defined over price/quality bundles $(p, q)$. All the consumers have the same reservation utility $U_{R}$. They all have unit demand and total demand is normalised to 1 .

With two firms, consumers search for the best price/quality combination - the one that maximises $U(p, q)$. As explained in the Introduction, we

\footnotetext{
${ }^{3} \mathrm{~A}$ more general function, if it is concave, should deliver the same qualitative results.
} 
consider three groups of consumers: (i) fully informed consumers know the prices and quality, (ii) partially informed consumers know the prices but not the quality, and (iii) fully uninformed consumers know neither price nor quality. These groups are referred to as $\mathrm{H}, \mathrm{M}$ and $\mathrm{L}$ consumers, respectively. The relative sizes of the first, second and third group are given by $\lambda_{H}, \lambda_{M}$ and $\lambda_{L}$ with

$$
1=\lambda_{H}+\lambda_{M}+\lambda_{L}
$$

A professional customer, who knows the firms and the product and hence can check costlessly for the prices and quality offered would belong to the $\mathrm{H}$ group. A new customer or a customer who lacks certain expertise to assess the quality, but who can take his time to check for different price offers, will belong to the $\mathrm{M}$ group. A person with substantially high alternative costs of searching for a better price and quality would belong to the L group.

Consumers search for the best offer. $\mathrm{H}$ consumers know exactly what the offers are and know how the utility from the best offer compares against their reservation utility $U_{R}$. M and $\mathrm{L}$ consumers search for the best offer based on their expectations and so they can not judge how the offer they select actually compares against their reservation utility, at least, during the search process. We assume that each consumer can learn the price and quality of the product at the moment of purchase. ${ }^{4}$ With this assumption, if a firm chooses $(p, q)$ such that $U(p, q)<U_{R}$, no consumer will buy.

Let us now put the behaviour of the consumers into a more formal context. To do so we discuss what the relevant information sets are, we discuss the possible actions of different types of consumers and their resulting payoffs and we point out what strategies are sequentially rational. ${ }^{5}$

Recollect that the firms move first and they simultaneously choose their $(p, q)$ bundles and then the consumers search for the best offer. Let $\left(p_{i}, q_{i}\right)$ be the offer of firm $i$. A consumer of type $\mathrm{H}$ knows both offers in full detail, i.e. he knows $\left(p_{1}, q_{1}\right)$ and $\left(p_{2}, q_{2}\right)$. An $\mathrm{H}$ consumer has three possible actions: to buy from firm 1, to buy from firm 2 and not to buy at all. The corresponding payoffs are $U\left(p_{1}, q_{1}\right), U\left(p_{2}, q_{2}\right)$ and $U_{R}$. Trivially, a sequentially rational strategy is to choose the action that gives the highest payoff.

A consumer of type $\mathrm{M}$ only knows $p_{1}$ and $p_{2}$ but not $q_{1}$ or $q_{2}$. Consequently, his information consists of all $\left(P_{1}, Q_{1}\right)$ and $\left(P_{2}, Q_{2}\right)$ bundles such

\footnotetext{
${ }^{4}$ This assumption is not crucial for our results. We focus on how consumers select between the firms and on the resulting competition. We do not focus on mechanisms that prevent the offers that breech the reservation utility from appearing on the market. So, we make the least complicated assumption that partially or fully uninformed consumers can learn the price and quality when they purchase. This is indeed true for the price. As for the quality, assessment costs are arguably lower once it is possible, for example, to test the good when being in the shop compared to learning the quality from an advertisement.

${ }^{5}$ See Kreps and Wilson (1982) for the definition of sequentially rational strategies. Roughly speaking they are like subgame perfect strategies but also for cases where a proper subgame can not be defined.
} 
that $P_{1}=p_{1}$ and $P_{2}=p_{2}$. We say that an $\mathrm{M}$ consumer has certain beliefs about the distribution of nodes in his information set. But we are not interested in his beliefs directly, rather we are interested in the utility he expects to get from either offer according to his beliefs. We use the notation $\hat{U}_{j}\left(p_{i} ; p_{-i}\right)$ to denote consumer $j$ 's expected utility from the offer of firm $i$ given both the price of firm $i$ and the price of its competitor, firm $-i$.

At this point an $\mathrm{M}$ consumer faces the following possible actions: go to firm 1, go to firm 2, do not buy at all. The corresponding expected payoffs are $\hat{U}_{j}\left(p_{1} ; p_{2}\right), \hat{U}_{j}\left(p_{2} ; p_{1}\right)$ and $U_{R}$. If he goes, say to firm $i$, he learns quality $q_{i}$. Now he knows what utility he will get if he buys and he can decide whether to buy or not. The corresponding payoffs are $U\left(p_{i}, q_{i}\right)$ and $U_{R}$. Trivially, a sequentially rational strategy is to choose in both decision nodes the actions that deliver the highest (expected) payoff.

Consumers of type $\mathrm{L}$ are those consumers who just go to a shop and buy if the product is not too bad for its price. L type consumers can be loyal customers or people with sufficiently high search costs. To model their behaviour we say that an L consumer can go to only one of the firms and we assume that a half of them goes to firm 1. Once in a shop an L consumer learns the price and quality of the product and he can either buy the product or leave the shop. Trivially, a sequentially rational strategy for him is to buy if $U\left(p_{i}, q_{i}\right) \geq U_{R}$.

For all consumers we assume that if they are indifferent between buying and not buying they buy, and if they are indifferent between going to firm 1 and firm 2 half of them goes to firm 1 and the other half to firm 2 .

The subsequent analysis is based on the assumption that utility function $U(p, q)$ is well-behaved.

Assumption 1. The utility function $U(p, q)$ is strictly decreasing in $p$, strictly increasing in $q$, strictly quasi-concave in $(p, q)$, twice differentiable in $(p, q)$. Moreover, $U(p, q)$ is such that the optimisation problem

$$
\max _{p, q} \Pi(p, q) \quad \text { s.t. } \quad U(p, q) \geq x
$$

has a solution for any $x \geq U_{R}$ (in a sense, the utility function should be sufficiently quasi-concave).

\subsection{Equilibria}

We concentrate on the role that prices have in signalling quality. In general, a certain price may signal a specific distribution of quality. ${ }^{6}$ But we would like to restrict our attention to a case where a certain price signals a certain quality. This restriction, while still allowing us to answer the questions raised in the introduction, considerably simplifies the mathematics of the

\footnotetext{
${ }^{6}$ This happens if firms play some mixed strategies over a region in $(p, q)$ space.
} 
model. Also, for simplicity, we restrict our attention to symmetric equilibria. Formally, we restrict our attention to equilibria that satisfy conditions 1-3 listed below.

Condition 1. The strategies of the firms are symmetric, i.e $\mathbb{P}_{1} \equiv \mathbb{P}_{2}$.

Given this condition we will drop index $i$ wherever appropriate.

Recollect that $\operatorname{supp}(\mathbb{P}) \in \mathbb{R}^{2}$ stands for the support of the distribution that either firm plays, i.e. it gives all the $(p, q)$ bundles that a firm may possibly play according to its strategy. If we want a certain price to signal a certain quality then we need $\operatorname{supp}(\mathbb{P})$ to form a curve in $(p, q)$ space. We thus have

Condition 2. $\operatorname{supp}(\mathbb{P})=\left\{(p, q): p \in\left[p_{l}, p_{h}\right], q=\hat{q}(p)\right\}$

In the above condition $p_{l}$ and $p_{h}$ are some arbitrary bounds and $\hat{q}(p)$ is some arbitrary function of $p$. Later we will see that for there to be an equilibrium $p_{l}, p_{h}$ and $\hat{q}(p)$ have to satisfy certain conditions.

Finally, to avoid rather technical difficulties, we also impose

Condition 3. $\hat{q}(p)$ is continuously differentiable in $p$ over $\left[p_{l}, p_{h}\right]$.

It is important to note that conditions 1-3 restrict the set of equilibrium strategies. We impose no restrictions on off-the-equilibrium strategies, i.e. a firm can deviate to playing any possible $(p, q)$ bundles if it finds doing so profitable.

We shall use

Definition 1. Function $\hat{q}(p)$ is called an equilibrium curve.

and

Definition 2. A sequential equilibrium that meets conditions 1-3 is called an exact signalling equilibrium.

By restricting our attention to exact signalling equilibria we essentially focus on equilibria in mixed strategies. In general, when the above conditions are not imposed, there are no pure strategy equilibria. ${ }^{7}$

This is a game with complete but imperfect information. So, we use the notion of sequential equilibrium (Kreps and Wilson (1982)). In short, players' strategies and beliefs form a sequential equilibrium if the strategies are sequentially rational given the beliefs and the beliefs are consistent with

\footnotetext{
${ }^{7}$ The formal proof is given in Dubovik (2007). The reason for the non-existence of pure strategy equilibria is essentially the same as in Varian (1980), namely that if a firm sticks to sell a good of a certain quality for a certain price, its rival finds it optimal to offer a slightly better deal for consumers.
} 
the strategies. As above we have discussed the sequentially rational strategies of consumers, it remains to discuss the consistency of beliefs and the sequential rationality of firms' strategies.

To turn to the consistency of beliefs, recollect that $\hat{U}_{j}\left(p_{i} ; p_{-i}\right)$ is an expectation of a partially informed consumer $j$ about the utility that he gets if he chooses to go to firm $i$. This expectation is consistent with the strategies of the firms if it is computed under the probability measure that defines those strategies. So,

$$
\hat{U}_{j}\left(p_{i} ; p_{-i}\right)=\mathbb{E}\left(\max \left(U(P, Q), U_{R}\right) \mid P=p_{i}\right) \quad \forall p_{i} \in\left[p_{l}, p_{h}\right],
$$

where $\mathbb{E}$ denotes an expectation under measure $\mathbb{P}$.

We take the maximum of $U(P, Q)$ and $U_{R}$ because, once in a shop, a consumer can choose not to buy if the realisation of $U(P, Q)$ is smaller than his reservation utility $U_{R}$.

Equation 4 tells us that consistent beliefs are the same for all consumers and do not depend upon the price of the rival firm given that these are onthe-equilibrium beliefs, i.e. if $p_{i} \in\left[p_{l}, p_{h}\right]$. In principle, off-the-equilibrium beliefs may have a more general form but we restrict attention to beliefs that have the same form as on-the-equilibrium beliefs:

$$
\hat{U}_{j}\left(p_{i} ; p_{-i}\right)=\hat{U}\left(p_{i}\right) .
$$

In an exact signalling equilibrium for any realisation of $P$ there is a unique corresponding realisation of $Q=\hat{q}(P)$. So, $U(P, Q)=U(P, \hat{q}(P))$ and

$$
\begin{aligned}
& \hat{U}(p)=\mathbb{E}\left(\max \left(U(P, Q), U_{R}\right) \mid P=p\right)= \\
& \mathbb{E}\left(\max \left(U(P, \hat{q}(P)), U_{R}\right) \mid P=p\right)=\max \left(U(p, \hat{q}(p)), U_{R}\right) .
\end{aligned}
$$

for all $p \in\left[p_{l}, p_{h}\right]$. Equation (6) gives the consistency condition for beliefs. Next, we turn to the sequential rationality of the strategies of the firms. We begin by writing down the expected profits a firm gets if it selects a particular $(p, q)$ bundle and if its rival is playing an equilibrium strategy (one that satisfies conditions 1-3).

Let $\mu_{H}(p, q), \mu_{M}(p)$ and $\mu_{L}$ denote the expected number of $\mathrm{H}$ type, $\mathrm{M}$ type and L type consumers that a firm gets if it charges $(p, q)$ such that $U(p, q) \geq U_{R}$. Given the sequentially rational strategies of consumers

$$
\mu_{H}(p, q)=\mathbb{P}(U(P, Q)<U(p, q)) \cdot \lambda_{H}+\mathbb{P}(U(P, Q)=U(p, q)) \cdot \frac{\lambda_{H}}{2}
$$

Indeed, all $\mathrm{H}$ consumers go to firm $i$ if its $(p, q)$ bundle gives higher utility than that of the rival. In general, a rival plays a mixed strategy and hence the chance to get all $\mathrm{H}$ consumers is given by $\mathbb{P}(U(P, Q)<U(p, q))$. If both offers give the same utility consumers split evenly. 
Next,

$$
\mu_{M}(p, q)=\mathbb{P}(\hat{U}(P)<\hat{U}(p)) \cdot \lambda_{M}+\mathbb{P}(\hat{U}(P)=\hat{U}(p)) \cdot \frac{\lambda_{M}}{2}
$$

Here the reasoning is similar to the one for $\mathrm{H}$ consumers. The only difference is that $\mathrm{M}$ consumers do not compare the actual utilities they get from either firm but rather they compare the expected utilities given the prices.

Finally, as was assumed,

$$
\mu_{L}=\frac{\lambda_{L}}{2}
$$

Define, for convenience,

$$
\mu(p, q)=\mu_{H}(p, q)+\mu_{M}(p)+\mu_{L} .
$$

Then expected profits are given by

$$
\pi(p, q)= \begin{cases}\mu(p, q) \cdot \Pi(p, q) & \text { if } U(p, q) \geq U_{R}, \\ 0 & \text { otherwise }\end{cases}
$$

As the firms choose simultaneously, a firm's sequentially rational strategy is simply a best response strategy. Choosing a $(p, q)$ bundle over an equilibrium curve $\hat{q}(p)$ is a best response strategy if and only if the profit function $\pi(p, q)$ attains its maximum along that equilibrium curve:

$$
\operatorname{supp}(\mathbb{P}) \in \arg \max _{p, q} \pi(p, q)
$$

If (12) is not satisfied for a given strategy (for a given $\mathbb{P}$ ), then the firm has an incentive to shift the probability mass from less profitable $(p, q)$ bundles to more profitable ones.

Equations (6) and (12) give necessary and sufficient conditions for there to be an exact signalling equilibrium. To have non-trivial results we make the following additional assumption:

Assumption 2. The model is non-degenerate, i.e. there exists $(p, q)$ such that $\Pi(p, q)>0$ and $U(p, q) \geq U_{R}$.

Since $\pi(p, q) \geq \mu_{L} \cdot \Pi(p, q)$ if $U(p, q) \geq U_{R}$, a firm can always guarantee itself some positive profits given the above assumption. So, in an equilibrium no firm will offer $(p, q)$ such that $U(p, q)<U_{R}$ because then it will have no consumers and zero profits. Consequently,

$$
\hat{U}(p)=\max \left(U(p, \hat{q}(p)), U_{R}\right)=U(p, \hat{q}(p)) \quad \forall p \in\left[p_{l}, p_{h}\right]
$$

We already know that in an exact signalling equilibrium $U(P, Q)=$ $U(P, \hat{q}(P))$. Using (13) then gives that

$$
\hat{U}(P)=U(P, \hat{q}(P))=U(P, Q)
$$


This result allows us to rewrite $\mu_{H}$ and $\mu_{M}$ in terms of a common distribution function. Let

$$
\begin{gathered}
F(u)=\mathbb{P}(U(P, Q)<u)=\mathbb{P}(\hat{U}(P)<u), \\
d F(u)=\mathbb{P}(U(P, Q)=u)=\mathbb{P}(\hat{U}(P)=u),
\end{gathered}
$$

where $d F$ is merely a notation. Then

$$
\begin{gathered}
\mu_{H}(p, q)=F(U(p, q)) \cdot \lambda_{H}+d F(U(p, q)) \cdot \frac{\lambda_{H}}{2}, \\
\mu_{M}(p)=F(\hat{U}(p)) \cdot \lambda_{M}+d F(\hat{U}(p)) \cdot \frac{\lambda_{M}}{2}
\end{gathered}
$$

To summarise, there is an exact signalling equilibrium if and only if

$$
\begin{aligned}
& \text { (i) } \quad \operatorname{supp}(\mathbb{P}) \in \arg \max _{(p, q)} \pi(p, q), \\
& \text { (ii) } \hat{U}(p)=U(p, \hat{q}(p)) \quad \forall p \in\left[p_{l}, p_{h}\right] .
\end{aligned}
$$

Later on we show that in any exact signalling equilibrium $U(p, \hat{q}(p))$ is strictly monotone in $p$. Therefore an exact signalling equilibrium is fully characterised by its equilibrium curve $\hat{q}(p)$, by the boundary points $p_{l}$ and $p_{h}$, by the distribution of utilities along the equilibrium curve, namely $F(u)$, and by its off-the-equilibrium beliefs, namely $\hat{U}(p)$ for $p \notin\left[p_{l}, p_{h}\right]$.

\section{Analysis}

In this section we solve for an exact signalling equilibrium, i.e. we solve for $F(u), \hat{q}(p)$ and $p_{l}, p_{h}$ given $U(p, q)$ and given the other parameters of the model. At first we assume that there exists an exact signalling equilibrium and we derive its properties. Later, we also discuss existence conditions for an exact signalling equilibrium.

\subsection{Utility Distribution}

One of the functions that characterises an exact signalling equilibrium is a CDF of utility over the equilibrium curve, namely $F(u)$. In this section we solve for $F(u)$.

Recollect that $F(u)=\mathbb{P}(U(P, \hat{q}(P))<u)$. Let us define

$$
\hat{U}(p)=U(p, \hat{q}(p)) .
$$

$\hat{U}(p)$ is continuous in $p$ because $U(p, q)$ is continuous in $p$ and $q$ and $\hat{q}(p)$ is differentiable. Therefore $\hat{U}(p)$ maps an interval into an interval. This allows us to define

$$
\left[U_{l}, U_{h}\right]=\hat{U}\left(\left[p_{l}, p_{h}\right]\right) .
$$


So, playing $(p, q)$ bundles along an equilibrium curve results into the corresponding utility being distributed over an interval.

We next show that $F(u)$ does not have atoms.

Lemma 1. $F(u)$ is continuous and $d F(u) \equiv 0$.

In economic terms, the chance that rivals provide the same utility level is zero. the next lemma argues that $U_{l}$ must be equal to $U_{R}$. The main reason is that the firm offering the worst utility only gets uninformed consumers and if $U_{l}>U_{R}$ he could make more profit by providing them a worse deal.

Lemma 2. $U_{l}=U_{R}$.

To find the functional form of $F(u)$ we need to be able to define equilibrium per-unit profits as a function of utility (theorem 1 will clarify why this is necessary). The following lemma allows us to do so.

Lemma 3. Given $u \in\left[U_{l}, U_{h}\right]$ per-unit profits $\Pi(p, \hat{q}(p))$ are the same for all $p \in \hat{U}^{-1}(u)$.

To understand this lemma, take an arbitrary $u$ from $\left[U_{l}, U_{h}\right]$. The isoutility curve corresponding to $u$ is implicitly given by $U(p, q)=u$. This iso-utility curve will intersect the equilibrium curve $\hat{q}(p)$ at least once. ${ }^{8}$ If $\left\{\left(p_{i}, \hat{q}\left(p_{i}\right)\right)\right\}$ is the set of intersection points, then $\left\{p_{i}\right\}$ is precisely $\hat{U}^{-1}(u)$ (recollect the definition of $\hat{U})$. At each intersection point $\left(p_{i}, \hat{q}\left(p_{i}\right)\right)$ we can compute the per-unit profits $\Pi\left(p_{i}, \hat{q}\left(p_{i}\right)\right)$. The lemma states that $\Pi\left(p_{i}, \hat{q}\left(p_{i}\right)\right)$ does not depend upon a particular choice of the intersection point, it only depends upon $u$. So, we use the notation $\hat{\Pi}(u)$ for such profits.

Formally, take an arbitrary $\tilde{p}(u)$ such that $\tilde{p}(u) \in \hat{U}^{-1}(u)$ for all $u \in$ $\left[U_{l}, U_{h}\right]$. Then

$$
\hat{\Pi}(u)=\Pi(\tilde{p}(u), \hat{q}(\tilde{p}(u))) .
$$

It is not possible to define $\hat{\Pi}(u)$ explicitly as it involves choosing a particular $\tilde{p}(u)$ and the functional form of $U(p, q)$ is not given. However, once a specific functional form of $U(p, q)$ is adopted, and once $\hat{q}(p)$ is known, it is possible to choose a particular $\tilde{p}(u)$ and hence solve for $\hat{\Pi}(u)$.

The last step before we solve for $F(u)$ is to simplify $\pi(p, q)$ :

Lemma 4. For $p \in\left[p_{l}, p_{h}\right]$ the profits are given by

$$
\pi(p, q)=\left(F(U(p, q)) \cdot \lambda_{H}+F(U(p, \hat{q}(p))) \cdot \lambda_{M}+\frac{\lambda_{L}}{2}\right) \cdot \Pi(p, q)
$$

if $U(p, q) \geq U_{R}$ and they equal 0 otherwise.

The result readily follows from lemma 1, from (20) (consistency of beliefs) and from the definition of $\pi(p, q)$.

Now we can solve for the functional form of $F(u)$.

\footnotetext{
${ }^{8}$ Later on we prove that only one intersection is possible.
} 


\section{Theorem 1.}

$$
F(u)=\frac{1}{2} \cdot \frac{\lambda_{L}}{\lambda_{H}+\lambda_{M}}\left(\frac{\hat{\Pi}\left(U_{R}\right)}{\hat{\Pi}(u)}-1\right) \quad \text { for } \quad u \in\left[U_{l}, U_{h}\right] .
$$

Proof. It follows from lemma 4 that

$$
\pi(p, \hat{q}(p))=\left(F(U(p, \hat{q}(p))) \cdot\left(\lambda_{H}+\lambda_{M}\right)+\frac{\lambda_{L}}{2}\right) \cdot \Pi(p, \hat{q}(p)) .
$$

Evaluating (24) at $\tilde{p}(u)$, noticing that $U(\tilde{p}(u), \hat{q}(\tilde{p}(u)))=u$ and using $(23)$ gives

$$
\pi(\tilde{p}(u), \hat{q}(\tilde{p}(u)))=\left(F(u) \cdot\left(\lambda_{H}+\lambda_{M}\right)+\frac{\lambda_{L}}{2}\right) \cdot \hat{\Pi}(u) .
$$

For there to be an equilibrium the strategies of the firms should be sequentially rational. This requirement was expressed in (19), we recollect it here:

$$
(p, \hat{q}(p)) \in \arg \max _{(\tilde{p}, \tilde{q})} \pi(\tilde{p}, \tilde{q})
$$

for all $p \in\left[p_{l}, p_{h}\right]$. Therefore $\pi(p, \hat{q}(p))$ is constant over this interval, denote its value by $\hat{\pi}$. Since $\tilde{p}(u) \in\left[p_{l}, p_{h}\right]$ we get

$$
\hat{\pi}=\left(F(u) \cdot\left(\lambda_{H}+\lambda_{M}\right)+\frac{\lambda_{L}}{2}\right) \cdot \hat{\Pi}(u) .
$$

By definition, $F\left(U_{l}\right)=0$. Also, $U_{l}=U_{R}$. So,

$$
\hat{\pi}=\frac{\lambda_{L}}{2} \cdot \hat{\Pi}\left(U_{R}\right)
$$

Plugging it back and solving for $F(u)$ gives the result.

It then follows that $U_{h}$ is implicitly given by $F\left(U_{h}\right)=1$. Using Theorem 1 it may alternatively be given by

$$
\hat{\Pi}\left(U_{h}\right)=\frac{1 / 2 \cdot \lambda_{L} \cdot \hat{\Pi}\left(U_{R}\right)}{\lambda_{H}+\lambda_{M}+1 / 2 \cdot \lambda_{L}} .
$$

\subsection{Equilibrium Curve}

The next function that characterises an exact signalling equilibrium is the equilibrium curve $\hat{q}(p)$. We solve for it now.

Theorem 2. If there is an exact signalling equilibrium then $\hat{q}(p)$ has to satisfy

$$
\frac{d \hat{q}}{d p}=-\frac{\lambda_{H}+\lambda_{M}}{\lambda_{M}} \cdot \frac{U_{p}^{\prime}(p, \hat{q}(p))}{U_{q}^{\prime}(p, \hat{q}(p))}-\frac{\lambda_{H}}{a \lambda_{M}}
$$

everywhere on $\left(p_{l}, p_{h}\right)$. 
Proof. It should be that

$$
\left.\frac{\partial \pi(p, q)}{\partial p}\right|_{(\tilde{p}, \hat{q}(\tilde{p}))}=0,\left.\quad \frac{\partial \pi(p, q)}{\partial q}\right|_{(\tilde{p}, \hat{q}(\tilde{p}))}=0
$$

because otherwise a firm may get higher profits by deviating along the gradient vector. Using lemma 4 we get

$$
\begin{array}{r}
\left.\frac{\partial \pi(p, q)}{\partial p}\right|_{(\tilde{p}, \hat{q}(\tilde{p}))}=\left(F^{\prime}(U(p, q)) \cdot U_{p}^{\prime}(p, q) \cdot \lambda_{H}+\right. \\
\left.F^{\prime}(U(p, \hat{q}(p))) \cdot\left(U_{p}^{\prime}(p, \hat{q}(p))+U_{q}^{\prime}(p, \hat{q}(p)) \cdot \hat{q}^{\prime}(p)\right) \cdot \lambda_{M}\right) \cdot(p-a q)+ \\
\left.\left(F(U(p, q)) \cdot \lambda_{H}+F(U(p, \hat{q}(p))) \cdot \lambda_{M}+\frac{\lambda_{L}}{2}\right) \cdot 1\right|_{(\tilde{p}, \hat{q}(\tilde{p}))}= \\
F^{\prime}(U(\tilde{p}, \hat{q}(\tilde{p})))\left(U_{p}^{\prime}(\tilde{p}, \hat{q}(\tilde{p}))\left(\lambda_{H}+\lambda_{M}\right)+U_{q}^{\prime}(\tilde{p}, \hat{q}(\tilde{p})) \cdot \hat{q}^{\prime}(\tilde{p}) \cdot \lambda_{M}\right) \cdot \\
(\tilde{p}-a \hat{q}(\tilde{p}))+\left(F\left(U(\tilde{p}, \hat{q}(\tilde{p})) \cdot\left(\lambda_{H}+\lambda_{M}\right)+\frac{\lambda_{L}}{2}\right)=0\right.
\end{array}
$$

and

$$
\begin{array}{r}
\left.\frac{\partial \pi(p, q)}{\partial q}\right|_{(\tilde{p}, \hat{q}(\tilde{p}))}=\left(F^{\prime}(U(p, q)) \cdot U_{q}^{\prime}(p, q) \cdot \lambda_{H}\right) \cdot(p-a q)+ \\
\left.\left(F(U(p, q)) \cdot \lambda_{H}+F(U(p, \hat{q}(p))) \cdot \lambda_{M}+\frac{\lambda_{L}}{2}\right) \cdot(-a)\right|_{(\tilde{p}, \hat{q}(\tilde{p}))} \\
\left(F^{\prime}(U(\tilde{p}, \hat{q}(\tilde{p}))) \cdot U_{q}^{\prime}(\tilde{p}, \hat{q}(\tilde{p})) \cdot \lambda_{H}\right) \cdot(\tilde{p}-a \hat{q}(\tilde{p}))+ \\
\left(F\left(U(\tilde{p}, \hat{q}(\tilde{p})) \cdot\left(\lambda_{H}+\lambda_{M}\right)+\frac{\lambda_{L}}{2}\right) \cdot(-a)=0\right.
\end{array}
$$

From (31)-(32) it follows after some algebra that

$$
\frac{d \hat{q}}{d \tilde{p}}=-\frac{\lambda_{H}+\lambda_{M}}{\lambda_{M}} \cdot \frac{U_{p}^{\prime}(\tilde{p}, \hat{q}(\tilde{p}))}{U_{q}^{\prime}(\tilde{p}, \hat{q}(\tilde{p}))}-\frac{\lambda_{H}}{a \lambda_{M}}
$$

What does the resulting $\hat{q}(p)$ look like? Figure 1 gives the answer. It depicts an equilibrium curve, iso-utility curves and isolines of per-unit profits.

To see why an equilibrium curve has a shape as given in the figure, rewrite the differential equation for $\hat{q}(p)$ as follows:

$$
\frac{d \hat{q}}{d p}=\frac{\lambda_{H}}{\lambda_{M}}\left(-\frac{U_{p}^{\prime}(p, \hat{q}(p))}{U_{q}^{\prime}(p, \hat{q}(p))}-\frac{1}{a}\right)-\frac{U_{p}^{\prime}(p, \hat{q}(p))}{U_{q}^{\prime}(p, \hat{q}(p))}
$$

and recall that the line representing $\bar{\Pi}$ has a slope equal to $1 / a$. The slope of the iso-utility curves is $-\frac{U_{p}^{\prime}(p, q)}{U_{q}^{\prime}(p, q)}$. Therefore it follows from (34) that if 
Figure 1: Equilibrium Curve

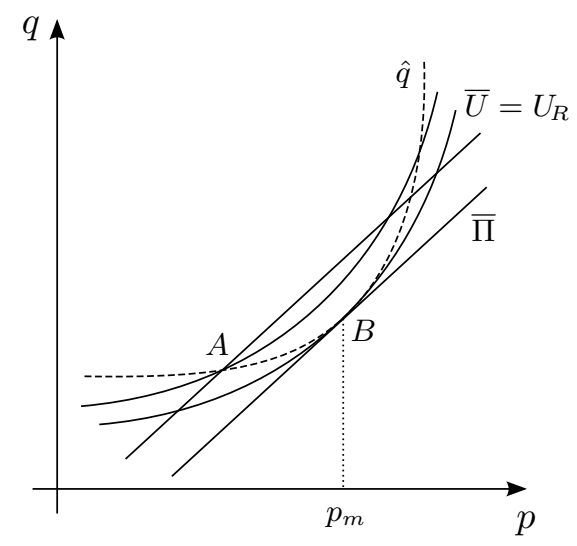

Notation: $\bar{X}$ stands for $X(p, q)=$ const, where constant is arbitrary; $\bar{X}=X_{0}$ stands for $X(p, q)=X_{0}$, where $X_{0}$ is some specific value.

the slope of an iso-utility curve is less than $1 / a$ (point $A$, for example), then the slope of an equilibrium curve is even smaller at that point and vice versa. If the slope is exactly $1 / a$ (point $B$ ), then an iso-utility curve and an equilibrium curve are tangent to each other and they are also tangent to an isoline of per-unit profits at that point. Therefore an equilibrium curve relative to iso-utility curves should look as depicted in the figure.

According to lemma 2 the lowest attainable utility along an equilibrium curve equals $U_{R}$, thus the equilibrium curve in the figure "lies" on an isoutility curve that corresponds to $U=U_{R}$.

\subsection{Boundary Points}

Earlier we have established a functional form of an equilibrium curve as well as a distribution of $(p, q)$ bundles over it. It remains to find out the boundary points of an equilibrium curve, namely $p_{l}$ and $p_{h}$. To address this problem, let us refer to figure 1 once more.

An equilibrium curve spans $\left[p_{l}, p_{h}\right]$, by definition. The figure shows that a choice of $\left[p_{l}, p_{h}\right]$ has important economic consequences: if $\left[p_{l}, p_{h}\right]$ is located to the left of the point of tangency $p_{m}$ then $U(p, \hat{q}(p))$ is decreasing in $p$, i.e. lower prices signal higher utility. If on the contrary, $\left[p_{l}, p_{h}\right]$ is to the right of $p_{m}$ then $U(p, \hat{q}(p))$ is increasing in $p$ and higher prices signal higher utility.

We find that both cases are possible depending upon the properties of $U(p, q)$, but that in both cases $p_{m}$ is the boundary point of the price interval. We also formally show that $U(p, \hat{q}(p))$ is indeed decreasing in $p$ in one case and increasing in $p$ in the other case. To state the main result, we need two 
definitions.

First, we formally define point $\left(p_{m}, q_{m}\right):^{9}$

Definition 3. Let $\left(p_{m}, q_{m}\right)$ be uniquely defined by

$$
\left(p_{m}, q_{m}\right)=\arg \max _{(p, q): U(p, q) \geq U_{R}} \Pi(p, q)
$$

Lemma A.1 (appendix) shows that the point $\left(p_{m}, q_{m}\right)$ defined in this way belongs to the equilibrium curve $\hat{q}(p)$ and that the equilibrium utility $U(p, \hat{q}(p))$ attains its minimum at $p_{m}$ - just like it is in the figure.

Second, we define a contract curve in the usual way as a curve that consists of all Pareto-efficient allocations in $(p, q)$ plane:

Definition 4. Let

$$
\left(p^{*}(x), q^{*}(x)\right)=\arg \max _{(p, q): U(p, q) \geq x} \Pi(p, q) .
$$

Then, if there exists a function $g$ such that $q^{*}(x)=g\left(p^{*}(x)\right)$, we shall refer to this function as a contract curve.

As, in principle, it is not necessary that $g(p)$ is defined for every $p$, we make the following technical assumption:

Assumption 3. A contract curve $g(p)$ is defined in the neighbourhood of $p_{m}$ and is differentiable at this point.

Now we can state the following theorem:

Theorem 3. If $g^{\prime}\left(p_{m}\right)<\frac{1}{a}$ and if there exists an exact signalling equilibrium then $\left[p_{l}, p_{h}\right]=\left[p_{l}, p_{m}\right]$ and $U(p, \hat{q}(p))$ is strictly decreasing in $p$ over this interval. Hence in such an equilibrium higher prices signal lower utility.

If $g^{\prime}\left(p_{m}\right)>\frac{1}{a}$ and if there exists an exact signalling equilibrium then $\left[p_{l}, p_{h}\right]=\left[p_{m}, p_{h}\right]$ and $U(p, \hat{q}(p))$ is strictly increasing in $p$ over this interval. Hence in such an equilibrium higher prices signal higher utility.

Theorem 3 tells there are two types of exact signalling equilibria with equilibrium utility $U(p, \hat{q}(p))$ being monotone in $p$ for either type. However, the theorem says nothing about monotonicity of the equilibrium quality, which is $\hat{q}(p)$. It turns out that $\hat{q}(p)$ is not necessarily monotone. Figure 2, which illustrates a particular exact signalling equilibrium, gives an example of a non-monotone curve. ${ }^{10}$

\footnotetext{
${ }^{9}$ This definition is valid. Indeed, the solution to the optimisation problem exists by assumption 1. Moreover, the solution is unique because $U(p, q)$ is strictly quasi-concave by the same assumption.

${ }^{10}$ We used the following example to build the figure: $U(p, q)=(q-1)^{1 / 2}(10-p)^{1 / 2}$, $\lambda_{H}=0.05, \lambda_{M}=0.1, \lambda_{L}=0.85, U_{R}=1$ and $a=1$. Section 4 gives another example and shows how to solve for an exact signalling equilibrium. If to apply that procedure to this example, one will get precisely fig. 2. However, this particular example we do not discuss in detail as the computations are harder comparing with the example of section 4 .
} 
Figure 2: Non-monotonic $\hat{q}(p)$

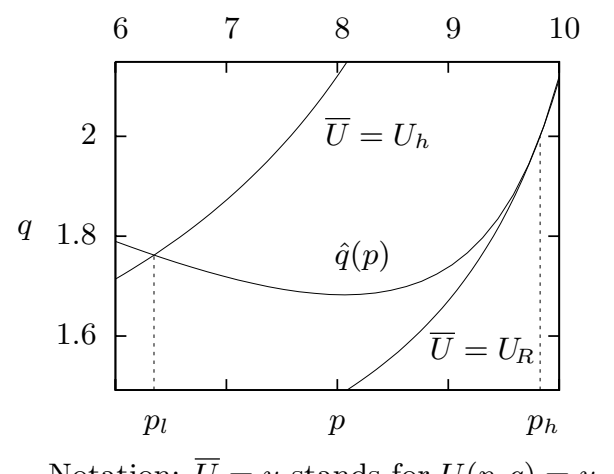

Notation: $\bar{U}=u$ stands for $U(p, q)=u$.

If the marginal utility of quality declines as the price increases, i.e., if $U_{q p}<0$, then $g(p)$ has a negative slope and $\left[p_{l}, p_{h}\right]=\left[p_{l}, p_{m}\right]$, so an equilibrium where lower prices signal higher utility results.

Given Theorem 3 we can solve for the boundary points $p_{l}$ and $p_{h}$. If $g^{\prime}\left(p_{m}\right)<\frac{1}{a}$ then $p_{h}=p_{m}$. As for $p_{l}$, recollect that $\left[U_{l}, U_{h}\right]=\hat{U}\left(\left[p_{l}, p_{h}\right]\right)$. But $\hat{U}(p)=U(p, \hat{q}(p))$ is strictly decreasing in $p$ when $g^{\prime}\left(p_{m}\right)<\frac{1}{a}$, therefore

$$
p_{l}=\hat{U}^{-1}\left(U_{h}\right) \text {. }
$$

Similarly, if $g^{\prime}\left(p_{m}\right)>\frac{1}{a}, p_{l}=p_{m}$ and

$$
p_{h}=\hat{U}^{-1}\left(U_{h}\right) \text {. }
$$

\subsection{Existence and Uniqueness}

In the previous sections we have uniquely determined all the parameters of an exact signalling equilibrium (except for off-the-equilibrium beliefs). Thefore we have the following theorem:

Theorem 4. There is at most one exact signalling equilibrium(up to offthe-equilibrium beliefs).

Proof. To do the proof we merely have to recollect some of the results obtained so far. $\left(p_{m}, q_{m}\right)$ was uniquely defined in def. 3. $\hat{q}(p)$ is given by a differential equation of theorem 2 and it is known to go through $\left(p_{m}, q_{m}\right)$ (lemma A.1), $\left(p_{m}, q_{m}\right)$ is thus the boundary point to uniquely solve the differential equation. Depending upon the sign of $g^{\prime}\left(p_{m}\right)$ we know that an exact signalling equilibrium spans either $\left[p_{l}, p_{m}\right]$ or $\left[p_{m}, p_{h}\right]$. In either case $U(p, \hat{q}(p))$ is strictly monotone (theorem 3 ) and therefore $\tilde{p}(u)$ is uniquely determined by $U(\tilde{p}(u), \hat{q}(\tilde{p}(u)))=u$. In turn, $\tilde{p}(u)$ gives us $\hat{\Pi}(u)$ and $\hat{\Pi}(u)$ gives $F(u)$ (see eq. (23) and theorem 1 respectively). Equation $F\left(U_{h}\right)=1$ uniquely determines $U_{h}$ and from $U_{h}$ we can determine the remaining $p_{l}$ (or 
$\left.p_{h}\right)$. Hence we can uniquely determine the parameters of an exact signalling equilibrium, namely $\hat{q}(p), p_{l}, p_{h}$ and $F(u)$.

This theorem is silent, however, about the existence of such equilibria. Now we want to address the existence issue. ${ }^{11}$ In the following sections we present an example where an equilibrium exists and another example where it does not. This leads to the question whether we can formulate parameter restrictions guaranteeing the existence of an exact signalling equilibrium. Since we consider a rather general $U(p, q)$ and since $\pi(p, q)$ is derived from $U(p, q)$ in a rather complicated way it renders to be very difficult (if not impossible) to provide such an existence result. However, we can address the question of existence from a different angle: given an arbitrary equilibrium curve $\hat{q}(p)$, can we find such parameters of our model that there is a corresponding exact signalling equilibrium, i.e. one that has $\hat{q}(p)$ as its equilibrium curve? Theorem 5 provides the answer.

We first have to discuss one basic requirement, though: an equilibrium curve should allow for positive per-unit profits as otherwise there can be no corresponding equilibrium.

Consider an arbitrary strictly increasing, strictly convex and twice differentiable equilibrium curve $\hat{q}(p)$ defined over some $\left[p_{l}, p_{h}\right]$. If we are looking for a corresponding equilibrium where lower prices signal higher utility, then $p_{m}=p_{h}$ and, consequently, $\hat{q}^{\prime}\left(p_{h}\right)=\frac{1}{a}$. Hence, we know $a$ and we can evaluate per-unit profits $\Pi(p, q)$. If there is an equilibrium it should be that

$$
\Pi(p, \hat{q}(p))=p-a \hat{q}(p)=p-\frac{\hat{q}(p)}{\hat{q}^{\prime}\left(p_{h}\right)}>0
$$

for all $p \in\left[p_{l}, p_{h}\right]$. Indeed, if the inequality does not hold for some $p$, then some equilibrium per-unit profits are zero or negative and so are the expected profits then. But zero or negative expected profits cannot be supported in an equilibrium.

For a strictly increasing and strictly convex $\hat{q}(p)$ equilibrium per-unit profits $\Pi(p, \hat{q}(p))$ are strictly increasing in $p$ over $\left[p_{l}, p_{m}\right]$ and therefore $(37)$ is equivalent to

$$
\Pi\left(p_{l}, \hat{q}\left(p_{l}\right)\right)=p_{l}-\frac{\hat{q}\left(p_{l}\right)}{\hat{q}^{\prime}\left(p_{h}\right)}>0
$$

In a similar way, if we are looking for a corresponding equilibrium where

\footnotetext{
${ }^{11}$ The main issue here is the following. Note that for any given utility function $U(p, q)$ and given the rest of the parameters $\left(\lambda_{H}, \lambda_{M}, \lambda_{L}\right.$ and $\left.a\right)$ we can always find an equilibrium curve $\hat{q}(p)$, its boundary points $p_{l}$ and $p_{h}$ and the distribution of utility over that curve, namely $F(u)$. We also know that profit function $\pi(p, q)$ will be constant along $\hat{q}(p)$ as required. But none of the results obtained so far guarantees that $\pi(p, q)$ will attain its maximum over $\hat{q}(p)$ what is a necessary and sufficient condition for there to be an exact signalling equilibrium.
} 
higher prices signal higher utility, it should be that

$$
\Pi\left(p_{h}, \hat{q}\left(p_{h}\right)\right)=p_{h}-\frac{\hat{q}\left(p_{h}\right)}{\hat{q}^{\prime}\left(p_{l}\right)}>0
$$

Given these two conditions we can state the theorem.

Theorem 5. Consider an arbitrary strictly increasing, strictly convex and twice differentiable equilibrium curve $\hat{q}(p)$ defined over $\left[p_{l}, p_{h}\right]$ and satisfying (38) or (39) or both. Then there exist such a utility function $U(p, q)$ satisfying assumption 1 , such parameters $\left(U_{R}, \lambda_{H}, \lambda_{M}, \lambda_{L}, a\right)$ and such offthe-equilibrium beliefs that there will be a corresponding exact signalling equilibrium, i.e one that has $\hat{q}(p)$ as its equilibrium curve. If (38) is satisfied, then $U(p, q),\left(U_{R}, \lambda_{H}, \lambda_{M}, \lambda_{L}, a\right)$ and off-the-equilibrium beliefs can be chosen such that lower prices signall higher utility in the equilibrium. If (39) is satisfied, they can be chosen such that higher prices signall higher utility in the equilibrium. If both conditions are satisfied, both types of an equilibrium can be achieved.

In other words, it may not be possible to have an exact signalling equilibrium for any $U(p, q)$, but at least there will be such equilibria for as many different forms of $U(p, q)$ as to generate every possible strictly increasing, strictly convex equilibrium curve $\hat{q}(p)$ that allows for positive per-unit profits.

\subsection{Pareto-efficiency}

An allocation is Pareto-efficient in this model if an iso-utility curve is tangent to an isoline of per-unit profits. But considering fig. 1, one can see that it is not the case for any $(p, \hat{q}(p))$ with $p \in\left[p_{l}, p_{h}\right)$. Therefore, equilibrium allocations are almost surely Pareto-inefficient.

If there are no partially-informed consumers, the model simplifies to the "Model of Sales" of Varian (1980) with prices being replaced by utilities. I.e., it becomes a single dimension model and consequently all the equilibrium allocations will be Pareto-efficient. Therefore, the partially-informed consumers and the incentives they create for firms to signal quality with price is what brings Pareto-inefficiency. Fully uninformed consumers do not create Pareto-inefficiency on their own, they merely create a redistribution in welfare.

\section{Example}

In this section, we illustrate the nature of an exact signalling equilibrium by considering an example. Take

$$
U(p, q)=\frac{1}{2} \ln q-p, U_{R}=-2, \lambda_{H}=\lambda_{M}=\frac{1}{5}, \lambda_{L}=\frac{3}{5}, a=1
$$


We begin by solving for $\left(p_{m}, q_{m}\right)$. To do so we solve

$$
\max _{p, q} \Pi(p, q) \quad \text { s.t. } \quad U(p, q) \geq U_{R} .
$$

and obtain

$$
p_{m}=\frac{1}{2} \ln \frac{1}{2}-U_{R}=\frac{1}{2} \ln \frac{1}{2}+2, \quad q_{m}=\frac{1}{2}
$$

Next we shall select whether it's an equilibrium where higher prices signal lower utility or the one where higher prices signal higher utility. To do it we need to know $g^{\prime}\left(p_{m}\right)$. From (40) one can readily see that $q_{m}$ doesn't depend upon $U_{R}$ and hence contract curve $g(p)=q_{m}=\frac{1}{2}$. Therefore $g^{\prime}\left(p_{m}\right)=$ $0<\frac{1}{a}=1$ and we have to search for an equilibrium to the left of $p_{m}$, i.e. $\left[p_{l}, p_{h}\right]=\left[p_{l}, p_{m}\right]$ (see theorem 3 ).

Let us now find $\hat{q}(p)$. Plugging our utility and the parameters into the differential equation for $\hat{q}(p)$ (see theorem 2) gives

$$
\frac{d \hat{q}(p)}{d p}=4 \hat{q}(p)-1
$$

Solving it and using the boundary condition $\hat{q}\left(p_{m}\right)=q_{m}$ gives:

$$
\hat{q}(p)=e^{4 p-8}+\frac{1}{4}
$$

To find utility distribution $F(u)$ we need to know $\hat{\Pi}(u)$ and for that we need to find $\tilde{p}(u)$ such that $U(\tilde{p}(u), \hat{q}(\tilde{p}(u)))=u$. Writing down this latter expression gives

$$
\frac{1}{2} \ln \left(e^{4 \tilde{p}(u)-8}+\frac{1}{4}\right)-\tilde{p}(u)=u
$$

A little bit of algebra gives the solution:

$$
\tilde{p}(u)=\frac{1}{2} \ln \left(\frac{1}{2}\left(e^{2 u}-\sqrt{e^{4 u}-e^{-8}}\right)\right)+4
$$

Having (43) and (45) we therefore also have

$$
\hat{\Pi}(u)=\tilde{p}(u)-a \hat{q}(\tilde{p}(u))=\tilde{p}(u)-\hat{q}(\tilde{p}(u))
$$

and

$$
F(u)=\frac{1}{2} \cdot \frac{\lambda_{L}}{\lambda_{H}+\lambda_{M}}\left(\frac{\hat{\Pi}\left(U_{R}\right)}{\hat{\Pi}(u)}-1\right)=\frac{3}{4}\left(\frac{\hat{\Pi}\left(U_{R}\right)}{\hat{\Pi}(u)}-1\right)
$$

Given $F(u)$ we can find $U_{h}$ since $F\left(U_{h}\right)=1$. Define

$$
z=\frac{1}{2}\left(e^{2 U_{h}+4}-\sqrt{e^{4 U_{h}+8}-1}\right)
$$


Figure 3: Equilibrium Characteristic Functions
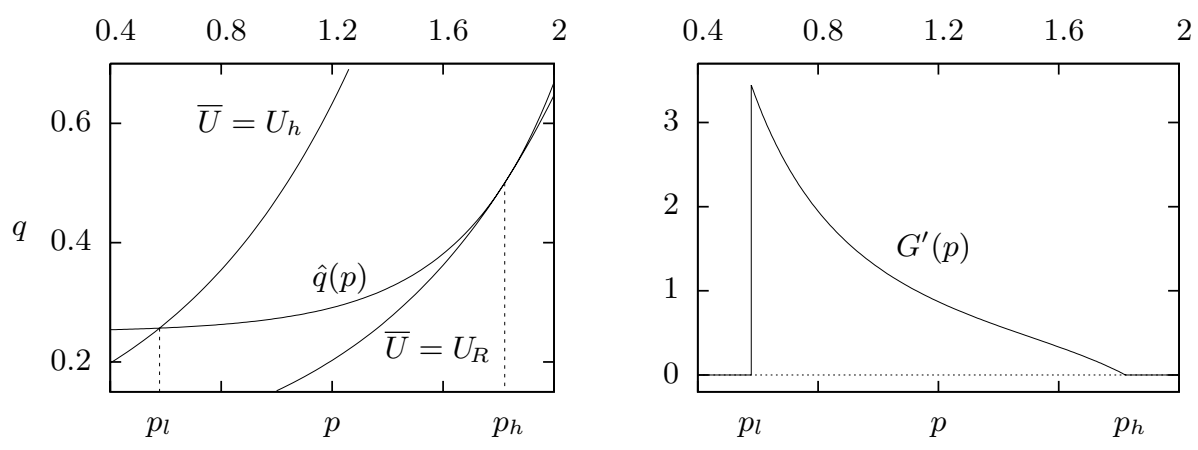

Notation: $\bar{U}=u$ stands for $U(p, q)=u$.

This way $z \leq \frac{1}{2}$ and $F\left(U_{h}\right)=1$ can be rewritten as

$$
\frac{1}{2} \ln z-z^{2}+\frac{7}{4}-\frac{3}{7} \hat{\Pi}\left(U_{R}\right)=0
$$

This equation can not be solved analytically but a numerical solution is very easy to get: $z \approx 0.08226$. Then, from the definition of $z$,

$$
U_{h}=\frac{1}{2} \ln \left(\frac{4 z^{2}+1}{4 z}\right)-2 \approx-1.43089
$$

Finally, $p_{l}=\tilde{p}\left(U_{h}\right) \approx 0.75109$.

Figure 3 plots a few important functions of our equilibrium candidate. The left plot gives $\hat{q}(p)$ together with iso-utility curves that correspond to $U_{l}=U_{R}$ and $U_{h}$. It is easy to see in the plot that higher prices signal lower utility in an equilibrium. In other words, if a partially informed consumer faces two products with different prices he will go for the cheapest product and, though the expected quality will be lower, the expected utility will be higher. The right plot gives the density function of the price distribution. Earlier we exclusively worked with utility distribution $F(u)$, but there is an easy transformation as

$$
\begin{aligned}
G(p)=\mathbb{P}(P<p)= & \mathbb{P}(U(P, \hat{q}(P))>U(p, \hat{q}(p)))= \\
1- & \mathbb{P}(U(P, \hat{q}(P)) \leq U(p, \hat{q}(p)))=1-F(U(p, \hat{q}(p))) .
\end{aligned}
$$

The right plot gives the density of this distribution, namely $G^{\prime}(p)$. From it we can see that the lower prices, lower quality occur more often than the higher prices, higher quality.

Recollect that $\pi(p, q)$ gives expected profits of one firm when the other firm is playing equilibrium strategies. Therefore for there to be an equilibrium it should be that $\pi(p, q)$ attains its maximum along the equilibrium curve $\hat{q}(p)$. Figure 4 plots $\pi(p, q)$. The left plot gives $2 \mathrm{D}$ slices of $\pi(p, q)$ for 
Figure 4: Equilibrium Profits
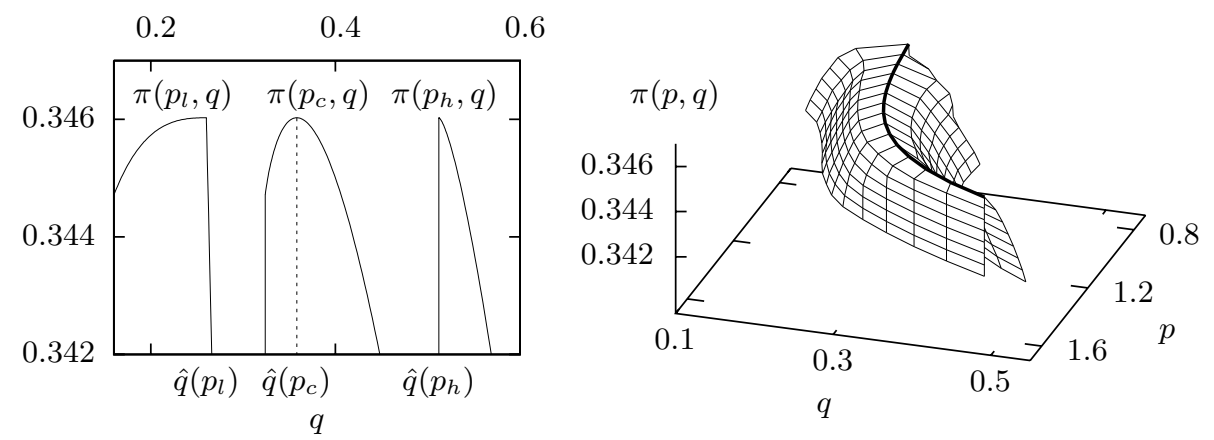

Comments: $p_{c}=\frac{1}{4} p_{l}+\frac{3}{4} p_{h}$ (the left plot); the bold line depicts $\pi(p, \hat{q}(p))$, i.e. the profits along the equilibrium curve (the right plot); also, for convenience, only a summit of $\pi(p, q)$ is shown in the right plot.

various $p$, the right plot attempts a $3 \mathrm{D}$ presentation. One can readily see that the condition in question is satisfied indeed and so we have an exact signalling equilibrium.

Does an exact signalling equilibrium always exist? Not necessary. Consider the same example but with $\lambda_{L}=\frac{1}{5}$ and $\lambda_{H}=\lambda_{M}=\frac{2}{5}$. It can be solved in the same way as before. Figure 5 gives the same plots as before but for this new example. We know that if there was an equilibrium it should have had the same $\pi(p, q)$ as we have found, but we have found $\pi(p, q)$ that does not have its maximum along the equilibrium curve. Hence we can conclude that there is no exact signalling equilibrium in this latter case.

\section{Conclusions}

We have considered a market where oligopolistic firms compete for consumers by varying prices and quality of their products and where consumers are heterogeneous in their knowledge of the prices and quality of the products offered: some know both the quality and prices, some know only the prices and some know neither. We have derived a signalling equilibrium for this setting that is characterised by firms playing a mixed strategy over a curve in a price-quality space. We have shown that this signalling equilibrium can be of two types. Both types are characterised by a dispersion of prices and quality and by Pareto-inefficiency of the price/quality offers. But in one type of equilibrium lower prices signal better price/quality ratios, while in the other type higher prices signal better price/quality ratios. Which type results depends on consumers' preferences: the cheapest offer is the best deal from a consumer perspective if the marginal utility of quality is declining in prices. 
Figure 5: Disequilibrium Profits
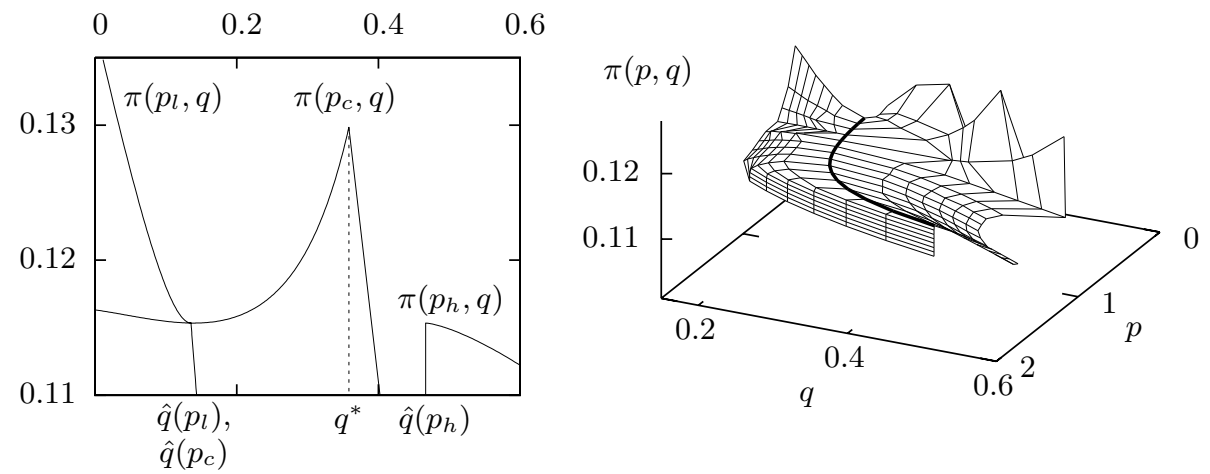

Comments: $p_{c}=\frac{4}{5} p_{l}+\frac{1}{5} p_{h}$ and $q^{*}$ is such that $U\left(p_{c}, q^{*}\right)=U_{h}$ (the left plot); the bold line depicts $\pi(p, \hat{q}(p))$, i.e. the profits along the equilibrium curve (the right plot); also, for convenience, only a summit of $\pi(p, q)$ is shown in the right plot; the sharp spokes to the back and right of the 3D picture are rendering artifacts - should a one steep "hill".

\section{References}

Kyle Bagwell and Michael H. Riordan. High and declining prices signal product quality. The American Economic Review, 81(1):224-239, 1991.

Damien Besancenot and Radu Vranceanu. Quality and price dispersion in an equilibrium search model. Journal of Economics and Business, 56(2): 99-116, 2004.

Yuk-Shee Chan and Hayne Leland. Prices and qualities in markets with costly information. The Review of Economic Studies, 49(4):499-516, 1982.

Russell Cooper and Thomas W. Ross. Prices, product qualities and asymmetric information: The competitive case. The Review of Economic Studies, 51(2):197-207, 1984.

Andrew F. Daughety and Jennifer F. Reinganum. Competition and confidentiality: Singaling quality in a duopoly when there is universal private information. Games and Economic Behavior, Forthcoming, 2004.

Andrei Dubovik. Oligopolistic competition in price and quality. Tinbergen Institute MPhil. thesis, 2007.

Mark N. Hertzendorf and Per Baltzer Overgaard. Prices as signals of quality in duopoly. URL http://www.econ.au.dk/vip_htm/povergaard/ pbohome/pbohome.html. 2001.

Maarten Janssen and Santanu Roy. Signaling quality through prices in an oligopoly. URL http://www.tinbergen.nl/discussionpapers/07081. pdf. Tinbergen Institute Discussion Paper, 2007. 
David M. Kreps and Robert Wilson. Sequential equilibria. Econometrica, 50(4):863-894, 1982.

William P. Rogerson. Price advertising and the deterioration of product quality. The Review of Economic Studies, 55(2):215-229, 1988.

Steven Salop and Joseph Stiglitz. Bargains and ripoffs: A model of monopolistically competitive price dispersion. The Review of Economic Studies, 44(3):493-510, 1977.

Alan Schwartz and Louis L. Wilde. Product quality and imperfect information. The Review of Economic Studies, 52(2):251-262, 1985.

George J. Stigler. The economics of information. The Journal of Political Economy, 69(3):213-225, 1961.

Hal R. Varian. A model of sales. The American Economic Review, 70(4): 651-659, 1980.

Asher Wolinksy. Prices as signals of product quality. The Review of Economic Studies, 50(4):647-658, 1983.

\section{Appendix}

Lemma 1. $F(u)$ is continuous and $d F(u) \equiv 0$.

Proof. For $u \in\left(-\infty, U_{l}\right) \cup\left(U_{h},+\infty\right)$ the cumulative distribution function $F(u)$ is continuous by definition. Consider now any point $\tilde{u} \in\left[U_{l}, U_{h}\right]$. Suppose $F(u)$ is discontinuous at $\tilde{u}$. Then $d F(\tilde{u})$ is strictly positive, i.e. a positive probability mass is assigned to an event $\{U(P, Q)=\tilde{u}\}$. This means that there is such $(p, q) \in \operatorname{supp}(\mathbb{P})$ that $U(p, q)=\tilde{u}$. Consider a situation when a firm deviates from $(p, q)$ to $(p, q+0)$. The expected number of partially uninformed consumers, $\mu_{M}$, and that of fully uninformed consumers, $\mu_{L}$, will not change as they do not depend upon $q$. Perunit profits will not change as well since $\Pi(p, q)$ is continuous in $q$ by definition. Consider $\mu_{H}$. At $(p, q+0)$

$$
\mu_{H}(p, q+0)=F(U(p, q+0)) \cdot \lambda_{H}+d F(U(p, q+0)) \frac{\lambda_{H}}{2} .
$$

$F(u)$ is a cumulative distribution function, therefore it has at most a countable number of points of discontinuity. Therefore $d F(\tilde{u}+0)=0 .{ }^{12}$ As $U(p, q)$ is strictly increasing in $q$ it holds that

$$
U(p, q+0)=U(p, q)+0=\tilde{u}+0 .
$$

\footnotetext{
${ }^{12} \mathrm{In}$ an $\varepsilon$-language the idea is to choose $\varepsilon>0$ small enough and also such that $d F(U(p, q+\varepsilon))=0$. Since the points of discontinuity can not cover an interval in full however small it is, it is always possible choose such an $\varepsilon$.
} 
Hence,

$$
d F(U(p, q+0))=0 .
$$

From the definition of $F(u)$ and $d F(u)$ it follows that

$$
F(\tilde{u}+0)=F(\tilde{u})+d F(\tilde{u}) .
$$

Plugging (54) and (55) into (52) gives

$$
\begin{aligned}
\mu_{H}(p, q+0)=[F(U(p, q))+ & d F(U(p, q))] \cdot \lambda_{H}> \\
& F(U(p, q)) \cdot \lambda_{H}+d F(U(p, q)) \frac{\lambda_{H}}{2}=\mu_{H}(p, q) .
\end{aligned}
$$

In section 2.3 we have shown that $U(p, q) \geq U_{R}$, so

$$
\pi(p, q)=\left(\mu_{H}(p, q)+\mu_{M}(p, q)+\mu_{L}(p, q)\right) \cdot \Pi(p, q) .
$$

Therefore $\pi(p, q+0)>\pi(p, q)$, what contradicts

$$
(p, q) \in \arg \max _{(\tilde{p}, \tilde{q})} \pi(\tilde{p}, \tilde{q}) .
$$

Therefore the supposition was wrong and $F(u)$ is continuous and $d F(u) \equiv 0$.

Lemma 2. $U_{l}=U_{R}$

Proof. From lemma 4 it follows that the profits are given by

$$
\pi(p, q)=\left(F(U(p, q)) \cdot \lambda_{H}+F(U(p, \hat{q}(p))) \cdot \lambda_{M}+\frac{\lambda_{L}}{2}\right) \cdot \Pi(p, q) .
$$

Consider $p \in\left[p_{l}, p_{h}\right]$ such that $U(p, \hat{q}(p))=U_{l}$. Such $p$ should exist because $U_{l}$ belongs to the support of $F(u)$ by definition. Also, by definition, $F\left(U_{l}\right)=0$. Therefore

$$
\pi(p, \hat{q}(p))=\frac{\lambda_{L}}{2} \cdot \Pi(p, \hat{q}(p)) .
$$

Clearly, $U_{l} \geq U_{R}$. Suppose that $U_{l}=U(p, \hat{q}(p))>U_{R}$. Since $\Pi(p, q)$ is strictly decreasing in $q$ and $U(p, q)$ is continuous in $q$, it is possible to choose such $\varepsilon>0$ that

$$
U(p, \hat{q}(p)-\varepsilon)>U_{R} \quad \text { and } \quad \Pi(p, \hat{q}(p)-\varepsilon)>\Pi(p, \hat{q}(p)) .
$$

Also, $F(U(p, \hat{q}(p)-\varepsilon))=0$ and therefore

$$
\pi(p, \hat{q}(p)-\varepsilon)=\frac{\lambda_{L}}{2} \cdot \Pi(p, \hat{q}(p)-\varepsilon)>\frac{\lambda_{L}}{2} \cdot \Pi(p, \hat{q}(p))=\pi(p, \hat{q}(p)) .
$$

This contradicts

$$
(p, \hat{q}(p)) \in \arg \max _{(\tilde{p}, \tilde{q})} \pi(\tilde{p}, \tilde{q})
$$

So, $U_{l}=U_{R}$

Lemma 3. Given $u \in\left[U_{l}, U_{h}\right]$ per-unit profits $\Pi(p, \hat{q}(p))$ are the same for all $p \in \hat{U}^{-1}(u)$. 
Proof. Take $p \in\left[p_{l}, p_{h}\right]$. Then it follows from lemma 4 that

$$
\pi(p, \hat{q}(p))=\left(F(\hat{U}(p)) \cdot\left(\lambda_{H}+\lambda_{M}\right)+\frac{\lambda_{L}}{2}\right) \cdot \Pi(p, \hat{q}(p)) .
$$

If there are different $p_{1}, p_{2}$ such that

$$
\hat{U}\left(p_{1}\right)=\hat{U}\left(p_{2}\right)=u
$$

then $\Pi\left(p_{1}, \hat{q}\left(p_{1}\right)\right)=\Pi\left(p_{1}, \hat{q}\left(p_{1}\right)\right)$. Indeed, if this is not the case, then equilibrium profits, i.e. the profits along an equilibrium curve, will differ between $p_{1}$ and $p_{2}$ as readily seen from (64). But profits have to attain their maximum along the equilibrium curve (see (19)) and hence they have to be constant along it as well.

Lemma A.1. $q_{m}=\hat{q}\left(p_{m}\right)$ and $p_{m} \in\left[p_{l}, p_{h}\right]$, i.e. point $\left(p_{m}, q_{m}\right)$ belongs to an equilibrium curve. Moreover, $U(p, \hat{q}(p))>U\left(p_{m}, \hat{q}\left(p_{m}\right)\right)$ for all $p \neq p_{m}$, i.e. point $\left(p_{m}, q_{m}\right)$ gives the minimum utility among all the points of an equilibrium curve.

Proof. Let $A=\left\{p \in\left[p_{l}, p_{h}\right] \mid U(p, \hat{q}(p))=U_{R}\right\}$. This way $A$ denotes the prices that, together with their equilibrium qualities, provide the lowest possible utility. Set $A$ is nonempty as follows from the definition of $U_{l}$ and from the result that $U_{l}=U_{R}$ (see lemma 2). Pick an arbitrary $p_{0} \in A$. Let $q_{0}=\hat{q}\left(p_{0}\right)$. Since $U\left(p_{0}, q_{0}\right)=U_{R}$ we have that $F\left(U\left(p_{0}, q_{0}\right)\right)=0$ and therefore

$$
\pi\left(p_{0}, q_{0}\right)=\frac{\lambda_{L}}{2} \Pi\left(p_{0}, q_{0}\right) .
$$

Consider now the following optimisation problem:

$$
\max _{p, q} \frac{\lambda_{L}}{2} \Pi(p, q) \quad \text { s.t. } \quad U(p, q) \geq U_{R} .
$$

Since a firm always attracts uninformed consumers while $U(p, q) \geq U_{R}$, the above problem gives the profits the firm can always guarantee to generate. We know that the unique solution to the problem is given by $\left(p_{m}, q_{m}\right)$. Since the solution is unique it should be that $\left(p_{0}, q_{0}\right)=\left(p_{m}, q_{m}\right)$. Indeed, if $\left(p_{0}, q_{0}\right) \neq\left(p_{m}, q_{m}\right)$ then the firms could generate strictly higher profits by deviating from $\left(p_{0}, q_{0}\right)$ to $\left(p_{m}, q_{m}\right)$ and then $\left(p_{0}, q_{0}\right)$ wouldn't be on an equilibrium curve, where it is by construction. This proves the first proposition of the lemma. To prove the second proposition it suffices to notice that since $\left(p_{0}, q_{0}\right)$ is uniquely defined, set $A$ consist of a single point, i.e. $A=\left\{\left(p_{0}, q_{0}\right)\right\}$.

Lemma A.2. $\frac{d}{d p} U(p, \hat{q}(p))>0$ for $p>p_{m}$ and $\frac{d}{d p} U(p, \hat{q}(p))<0$ for $p<p_{m}$.

Proof. For convenience let $\hat{U}$ stand for $U(p, \hat{q}(p))$ and let the same be for the derivatives, e.g. $\hat{U}_{p}$ stands for $U_{p}(p, \hat{q}(p))=\left.\frac{\partial U(p, q)}{\partial p}\right|_{(p, \hat{q}(p))}$ Suppose $\frac{d \hat{U}}{d p}<0$ at some point $p_{0}>p_{m}$. Utility function $U(p, q)$ and equilibrium curve $\hat{q}(p)$ are continuous by assumption, therefore $U(p, \hat{q}(p))$ is continuous. Also $U\left(p_{0}, \hat{q}\left(p_{0}\right)\right)>U\left(p_{m}, \hat{q}\left(p_{m}\right)\right)$ by lemma A.1 and $U(p, \hat{q}(p))$ is decreasing at point $p_{0}$ by the above supposition. Since $p_{0}>p_{m}$ we can then find $p_{1} \in\left(p_{m}, p_{0}\right)$ such that

$$
U\left(p_{1}, \hat{q}\left(p_{1}\right)\right)=U\left(p_{0}, \hat{q}\left(p_{0}\right)\right) \quad \text { and }\left.\quad \frac{d \hat{U}}{d p}\right|_{p_{1}}>0
$$


Let us expand $\frac{d \hat{U}}{d p}$ :

$$
\frac{d}{d p} U(p, \hat{q}(p))=\hat{U}_{p}+\hat{U}_{q} \frac{d \hat{q}}{d p}=\frac{\lambda_{H} \hat{U}_{q}}{\lambda_{M}}\left(-\frac{\hat{U}_{p}}{\hat{U}_{q}}-\frac{1}{a}\right)
$$

where the expression for $\hat{q}^{\prime}(p)$ comes from theorem 2. Using the above to rewrite $\left.\frac{d \hat{U}}{d p}\right|_{p_{0}}<0$ and $\left.\frac{d \hat{U}}{d p}\right|_{p_{1}}>0$ gives

$$
-\frac{U_{p}\left(p_{0}, \hat{q}\left(p_{0}\right)\right)}{U_{q}\left(p_{0}, \hat{q}\left(p_{0}\right)\right)}<\frac{1}{a} \quad \text { and } \quad-\frac{U_{p}\left(p_{1}, \hat{q}\left(p_{1}\right)\right)}{U_{q}\left(p_{1}, \hat{q}\left(p_{1}\right)\right)}>\frac{1}{a} .
$$

Let us now consider an iso-utility curve that goes through $\left(p_{0}, \hat{q}\left(p_{0}\right)\right)$ and $\left(p_{1}, \hat{q}\left(p_{1}\right)\right)$. It's the same iso-utility curve because $U\left(p_{0}, \hat{q}\left(p_{0}\right)\right)=U\left(p_{1}, \hat{q}\left(p_{1}\right)\right)$. Denote this curve by $\tilde{q}(p)$, i.e. $\tilde{q}(p)$ is implicitly defined by

$$
U(p, \tilde{q}(p))=U\left(p_{1}, \hat{q}\left(p_{1}\right)\right)=U\left(p_{0}, \hat{q}\left(p_{0}\right)\right) .
$$

This definition is valid since $U(p, q)$ is strictly increasing in $q$ and so there is only one solution for $\tilde{q}$ in the above equation. For the same reason

$$
\hat{q}\left(p_{0}\right)=\tilde{q}\left(p_{0}\right) \quad \text { and } \quad \hat{q}\left(p_{1}\right)=\tilde{q}\left(p_{1}\right) .
$$

Differentiating (71) gives

$$
\frac{d \tilde{q}}{d p}=-\frac{U_{p}(p, \tilde{q}(p))}{U_{q}(p, \tilde{q}(p))} .
$$

Bringing together (70), (72) and (73) gives

$$
\tilde{q}^{\prime}\left(p_{0}\right)<\frac{1}{a}<\tilde{q}^{\prime}\left(p_{1}\right) .
$$

At the same time $U(p, q)$ is strictly decreasing in $p$, strictly increasing in $q$ and strictly quasi-concave, therefore $\tilde{q}(p)$ is convex, i.e. $\tilde{q}^{\prime \prime}(p)>0$. It shall follow then that $\tilde{q}^{\prime}\left(p_{0}\right)>\tilde{q}^{\prime}\left(p_{1}\right)$ since $p_{0}>p_{1}$, but that contradicts (74). Therefore the earlier supposition that $\left.\frac{d \hat{U}}{d p}\right|_{p_{0}}<0$ is wrong. Suppose now that $\left.\frac{d \hat{U}}{d p}\right|_{p_{0}}=0$. From (69) it then follows that

$$
\hat{q}^{\prime}\left(p_{0}\right)=-\frac{U_{p}\left(p_{0}, \hat{q}\left(p_{0}\right)\right)}{U_{q}\left(p_{0}, \hat{q}\left(p_{0}\right)\right)}=\frac{1}{a} .
$$

Also,

$$
\tilde{q}^{\prime}\left(p_{0}\right)=-\frac{U_{p}\left(p_{0}, \tilde{q}\left(p_{0}\right)\right)}{U_{q}\left(p_{0}, \tilde{q}\left(p_{0}\right)\right)}=-\frac{U_{p}\left(p_{0}, \hat{q}\left(p_{0}\right)\right)}{U_{q}\left(p_{0}, \hat{q}\left(p_{0}\right)\right)}=\frac{1}{a} .
$$

Taking $\frac{d^{2} \hat{U}}{d p^{2}}$, considering it at point $p_{0}$ and plugging in the above expression for $\hat{q}^{\prime}\left(p_{0}\right)$ gives

$$
\left.\frac{d^{2} \hat{U}}{d p}\right|_{p_{0}}=\left.\frac{\lambda_{H}}{\lambda_{M}}\left(-U_{p p}-2 \frac{1}{a} U_{p q}-\frac{1}{a^{2}} U_{q q}\right)\right|_{\left(p_{0}, \hat{q}\left(p_{0}\right)\right)}
$$

Using (73) to get $\frac{d^{2}}{d p^{2}} \tilde{q}$, considering it at $p_{0}$ and plugging in the expression for $\tilde{q}^{\prime}\left(p_{0}\right)$ gives

$$
\left.\frac{d^{2}}{d p^{2}} \tilde{q}\right|_{p_{0}}=\left.\frac{1}{U_{q}}\left(-U_{p p}-2 \frac{1}{a} U_{p q}-\frac{1}{a^{2}} U_{q q}\right)\right|_{\left(p_{0}, \tilde{q}\left(p_{0}\right)\right)}
$$


But $\frac{d^{2}}{d p^{2}} \tilde{q}>0$ because iso-utility curves are convex, $U_{q}>0$ because $U(p, q)$ is strictly increasing in $q,\left(p_{0}, \tilde{q}\left(p_{0}\right)\right)=\left(p_{0}, \hat{q}\left(p_{0}\right)\right)$. Therefore $\left.\frac{d^{2} \hat{U}}{d p^{2}}\right|_{p_{0}}>0$. So, $\hat{U}$ is strictly convex at $p_{0}$ with $\left.\frac{d \hat{U}}{d p}\right|_{p_{0}}=0$. Consequently $\exists p_{2} \in\left(p_{m}, p_{0}\right):\left.\frac{d \hat{U}}{d p}\right|_{p_{2}}<0$. As was shown before this can not be the case and therefore the supposition that $\left.\frac{d \hat{U}}{d p}\right|_{p_{0}}=0$ is also wrong. Summarising both arguments gives that $\frac{d \hat{U}}{d p}>0$ for $p>p_{m}$. Analogues arguments give that $\frac{d \hat{U}}{d p}<0$ for $p<p_{m}$.

Lemma A.3. For any $p \neq p_{m}$

$$
\left.\frac{\partial^{2} \pi(p, q)}{\partial^{2} q}\right|_{(p, \hat{q}(p))}=\frac{a^{2} \lambda_{L} \lambda_{M}}{\lambda_{H}} \cdot \frac{\hat{\Pi}\left(U_{R}\right)}{\Pi(p, \hat{q}(p))}\left(\frac{1}{2} \frac{\hat{U}_{p q}-\frac{\hat{U}_{p}}{\hat{U}_{q}} \hat{U}_{q q}}{\hat{U}_{q}+a \hat{U}_{p}}+\frac{1}{\Pi(p, \hat{q}(p))}\right)
$$

where $\hat{U}_{p}=\left.\frac{\partial U(p, q)}{\partial p}\right|_{(p, \hat{q}(p))}$ and similarly for $\hat{U}_{q}, \hat{U}_{p q}$ and $\hat{U}_{q q}$.

Proof. We prove this lemma in a straightforward way. Recollect from lemma 4 that

$$
\pi(p, q)=\left(F(U(p, q)) \cdot \lambda_{H}+F(U(p, \hat{q}(p))) \cdot \lambda_{M}+\frac{\lambda_{L}}{2}\right) \cdot \Pi(p, q)
$$

with

$$
\Pi(p, q)=p-a q .
$$

Distribution function $F$ comes from theorem 1:

$$
F(u)=\frac{1}{2} \cdot \frac{\lambda_{L}}{\lambda_{H}+\lambda_{M}}\left(\frac{\hat{\Pi}\left(U_{R}\right)}{\hat{\Pi}(u)}-1\right) .
$$

If we are to differentiate $\pi(p, q)$ we need to know $\hat{q}^{\prime}(p)$ and $\hat{\Pi}^{\prime}(u)$. The former derivative we take from theorem 2 :

$$
\frac{d \hat{q}}{d p}=-\frac{\lambda_{H}+\lambda_{M}}{\lambda_{M}} \cdot \frac{U_{p}^{\prime}(p, \hat{q}(p))}{U_{q}^{\prime}(p, \hat{q}(p))}-\frac{\lambda_{H}}{a \lambda_{M}} .
$$

As for the latter derivative, recollect that

$$
\hat{\Pi}(u)=\Pi(\tilde{p}(u), \hat{q}(\tilde{p}(u)))
$$

where $\tilde{p}(u)$ could be any function such that $U(\tilde{p}(u), \hat{q}(\tilde{p}(u)))=u$. We'll be looking at the second order derivative of $\pi(p, q)$ at point $\left(p_{0}, \hat{q}\left(p_{0}\right)\right)$ of an equilibrium curve with $p_{0} \neq p_{m}$. For this point we can be more precise about $\tilde{p}(u)$. Indeed, from lemma A.2 we know that

$$
\frac{d}{d p} U(p, \hat{q}(p)) \neq 0 \quad \text { for } \quad p \neq p_{m}
$$

Also $U(p, \hat{q}(p))$ is twice differentiable because for $U(p, q)$ it was assumed and $\hat{q}(p)$ is itself defined by a differential equation that involves only differentiable functions. So, by an inverse function theorem there is a unique continuously differentiable $\tilde{p}(u)$ defined in the neighbourhood of $u_{0}=U\left(p_{0}, \hat{q}\left(p_{0}\right)\right)$ by

$$
U(\tilde{p}(u), \hat{q}(\tilde{p}(u)))=u
$$


with its derivative given by

$$
\begin{aligned}
\frac{d \tilde{p}(u)}{d u}=\frac{1}{U_{p}(\tilde{p}(u), \hat{q}(\tilde{p}(u))+} & U_{q}\left(\tilde{p}(u), \hat{q}(\tilde{p}(u)) \cdot \hat{q}^{\prime}(\tilde{p}(u))\right. \\
& \quad-\frac{a \lambda_{M}}{\lambda_{H}} \frac{1}{a \cdot U_{p}\left(\tilde{p}(u), \hat{q}(\tilde{p}(u))+U_{q}(\tilde{p}(u), \hat{q}(\tilde{p}(u))\right.} .
\end{aligned}
$$

Expressions (80), (81), (82), (83) and (86) allow one to calculate the second order derivative of $\pi(p, q)$ in $q$ in a straightforward way. Evaluating the resulting derivative at $\left(p_{0}, \hat{q}\left(p_{0}\right)\right)$, noticing that

$$
\tilde{p}\left(U\left(p_{0}, \hat{q}\left(p_{0}\right)\right)\right)=p_{0}
$$

and noticing that $p_{0}$ was chosen arbitrary just not to equal $p_{m}$ immediately gives the result of the lemma.

\section{Lemma A.4.}

$$
\begin{array}{llll}
U_{p q}\left(p_{m}, \hat{q}\left(p_{m}\right)\right)+\frac{1}{a} U_{q q}\left(p_{m}, \hat{q}\left(p_{m}\right)\right)<0 & \Rightarrow & {\left[p_{l}, p_{h}\right]=\left[p_{l}, p_{m}\right],} \\
U_{p q}\left(p_{m}, \hat{q}\left(p_{m}\right)\right)+\frac{1}{a} U_{q q}\left(p_{m}, \hat{q}\left(p_{m}\right)\right)>0 & \Rightarrow & {\left[p_{l}, p_{h}\right]=\left[p_{m}, p_{h}\right]}
\end{array}
$$

In other words, either the equilibrium lies in the segment to the left of $p_{m}$ where higher prices signal lower utility or the equilibrium lies in the segment to the right of $p_{m}$ where higher prices signal higher utility (assumption 3 guarantees that the case when $U_{p q}\left(p_{m}, \hat{q}\left(p_{m}\right)\right)+\frac{1}{a} U_{q q}\left(p_{m}, \hat{q}\left(p_{m}\right)\right)=0$ can not happen).

Proof. Consider the case when $U_{p q}\left(p_{m}, \hat{q}\left(p_{m}\right)\right)+\frac{1}{a} U_{q q}\left(p_{m}, \hat{q}\left(p_{m}\right)\right)<0$. Suppose $p_{h}>p_{m}$. Then we can consider the limit of $\left.\frac{\partial^{2} \pi(p, q)}{\partial^{2} q}\right|_{(p, \hat{q}(p))}$ as $p$ approaches $p_{m}$ from the right. To do so let us start with the limit of $-\frac{\hat{U}_{p}}{\hat{U}_{q}}$. By definition of $p_{m}$ and by lemma A.1

$$
-\frac{U_{p}\left(p_{m}, \hat{q}\left(p_{m}\right)\right)}{U_{q}\left(p_{m}, \hat{q}\left(p_{m}\right)\right)}=\frac{1}{a}
$$

By lemma A.2

$$
\frac{d}{d p} U(p, \hat{q}(p))>0 \quad \text { for } \quad p>p_{m} .
$$

Taking the derivative shows that this condition is equivalent to ${ }^{13}$

$$
-\frac{U_{p}(p, \hat{q}(p))}{U_{q}(p, \hat{q}(p))}>\frac{1}{a} \quad \text { for } \quad p>p_{m} .
$$

Moreover, $-\frac{\hat{U}_{p}}{\hat{U}_{q}}$ is continuous in $p$. Therefore we have that

$$
-\frac{\hat{U}_{p}}{\hat{U}_{q}} \rightarrow\left(\frac{1}{a}\right)^{+} \quad \text { as } \quad p \rightarrow p_{m}^{+}
$$

\footnotetext{
${ }^{13}$ See the mentioned lemma for more details.
} 
Hence

$$
\begin{aligned}
\left.\lim _{p \rightarrow p_{m}^{+}} \frac{\partial^{2} \pi(p, q)}{\partial^{2} q}\right|_{(p, \hat{q}(p))} & = \\
\lim _{p \rightarrow p_{m}^{+}} \frac{a^{2} \lambda_{L} \lambda_{M}}{\lambda_{H}} \cdot \frac{\hat{\Pi}\left(U_{R}\right)}{\Pi(p, \hat{q}(p))}\left(\frac{1}{2 a \hat{U}_{q}} \frac{\hat{U}_{p q}-\frac{\hat{U}_{p}}{\hat{U}_{q}} \hat{U}_{q q}}{\frac{1}{a}-\left(-\frac{\hat{U}_{p}}{\hat{U}_{q}}\right)}+\frac{1}{\Pi(p, \hat{q}(p))}\right) & = \\
& +\infty
\end{aligned}
$$

The sign comes from the preceding discussion and from the observation that $\hat{\Pi}\left(U_{R}\right)$, $\Pi(p, \hat{q}(p))$ and $U_{q}$ are all strictly positive. But (92) contradicts the necessary condition that $\left.\frac{\partial^{2} \pi(p, q)}{\partial^{2} q}\right|_{(p, \hat{q}(p))} \leq 0$ for all $p \in\left[p_{l}, p_{h}\right]$. Therefore if there is an exact signalling equilibrium it should be that $p_{h} \leq p_{m} \cdot{ }^{14}$ But $p_{m} \in\left[p_{l}, p_{h}\right]$ (lemma A.1), hence $p_{m}=p_{h}$. Analogous arguments hold for $U_{p q}\left(p_{m}, \hat{q}\left(p_{m}\right)\right)+\frac{1}{a} U_{q q}\left(p_{m}, \hat{q}\left(p_{m}\right)\right)>$ 0 .

\section{Lemma A.5.}

$$
U_{p q}\left(p_{m}, \hat{q}\left(p_{m}\right)\right)+\frac{1}{a} U_{q q}\left(p_{m}, \hat{q}\left(p_{m}\right)\right) \gtrless 0 \quad \Leftrightarrow \quad g^{\prime}\left(p_{m}\right) \gtrless \frac{1}{a}
$$

Proof. Writing down the necessary conditions for the optimisation problem that defines $g(p)$ gives

$$
-\frac{U_{p}(p, g(p))}{U_{q}(p, g(p))}=\frac{1}{a}
$$

Or, equivalently,

$$
a U_{p}(p, g(p))+U_{q}(p, g(p))=0
$$

Differentiating (94) in $p$ and rearranging the terms gives

$$
g^{\prime}(p)=-\frac{U_{p p}+\frac{1}{a} U_{q p}}{U_{p q}+\frac{1}{a} U_{q q}}=\frac{-U_{p p}-2 \frac{1}{a} U_{p q}-\frac{1}{a^{2}} U_{q q}}{U_{p q}+\frac{1}{a} U_{q q}}+\frac{1}{a}
$$

where $U_{p p}=\left.\frac{\partial^{2} U(p, q)}{\partial p^{2}}\right|_{(p, g(p))}$, etc. Consider now an iso-utility curve going through $\left(p_{m}, q_{m}\right)$. Namely, consider $\tilde{q}(p)$ defined by

$$
U(p, \tilde{q}(p))=U\left(p_{m}, q_{m}\right)
$$

Twice differentiating this expression, evaluating it at $\left(p_{m}, q_{m}\right)$, noticing that

$$
\tilde{q}^{\prime}\left(p_{m}\right)=-\frac{U_{p}\left(p_{m}, \tilde{q}\left(p_{m}\right)\right)}{U_{q}\left(p_{m}, \tilde{q}\left(p_{m}\right)\right)}=\frac{1}{a}
$$

due to the definition of $\left(p_{m}, q_{m}\right)$ and rearranging the terms gives

$$
\tilde{q}^{\prime \prime}\left(p_{m}\right)=\left.\frac{1}{U_{q}}\left(-U_{p p}-2 \frac{1}{a} U_{p q}-\frac{1}{a^{2}} U_{q q}\right)\right|_{\left(p_{m}, \tilde{q}\left(p_{m}\right)\right)}
$$

\footnotetext{
${ }^{14}$ In this case we can not consider a limit from the right and the contradiction doesn't hold.
} 
Iso-utility curves are strictly convex (assumption 1$)$, so $\tilde{q}^{\prime \prime}\left(p_{m}\right)>0$. Also, $\left(p_{m}, q_{m}\right)$ belongs to the contract curve $g(p)$, to the equilibrium curve $\hat{q}(p)$ and to the isoutility curve $\tilde{q}(p)$, so $q_{m}=g\left(p_{m}\right)=\hat{q}\left(p_{m}\right)=\tilde{q}\left(p_{m}\right)$. So, we can use $U_{p p}\left(p_{m}, g\left(p_{m}\right)\right)$, $U_{p p}\left(p_{m}, \hat{q}\left(p_{m}\right)\right)$ and $U_{p p}\left(p_{m}, \tilde{q}\left(p_{m}\right)\right)$ and the others interchangeably. But then the statement of the lemma readily follows from $(95),(98)$, from $\tilde{q}^{\prime \prime}\left(p_{m}\right)>0$ and from $U_{q}\left(p_{m}\right)>0$.

Theorem 3. If $g^{\prime}\left(p_{m}\right)<\frac{1}{a}$ and if there exists an exact signalling equilibrium then $\left[p_{l}, p_{h}\right]=\left[p_{l}, p_{m}\right]$ and $U(p, \hat{q}(p))$ is strictly decreasing in $p$ over this interval. Hence in an equilibrium higher prices signal lower utility.

And vice versa. If $g^{\prime}\left(p_{m}\right)>\frac{1}{a}$ and if there exists an exact signalling equilibrium then $\left[p_{l}, p_{h}\right]=\left[p_{m}, p_{h}\right]$ and $U(p, \hat{q}(p))$ is strictly increasing in $p$ over this interval. Hence in an equilibrium higher prices signal higher utility.

Proof. The formall proof is fully contained in lemmas A.1-A.5. Next we only give a bit of explanation. For there to be an equilibrium, profit function $\pi(p, q)$ should attain its maximum along an equilibrium curve $\hat{q}(p)$ or otherwise the firms will deviate from playing $(p, q)$ bundles over it. The idea of the proof is to apply second order necessary conditions to check whether $\pi(p, q)$ can indeed attain its maximum over $\hat{q}(p)$ given different choices of $p_{l}$ and $p_{h}$. So, we need $\pi(p, q)$ to be concave in the neighbourhood of each point of an equilibrium curve. In general we have to consider a Hessian to check that but for this proof it suffices and it is convenient to check concavity only in $q$, i.e. we look at the following second order necessary condition:

$$
\left.\frac{\partial^{2} \pi(p, q)}{\partial^{2} q}\right|_{(p, \hat{q}(p))} \leq 0 \quad \text { for } \quad p \in\left[p_{l}, p_{h}\right] .
$$

Lemma A.3 provides us with $\left.\frac{\partial^{2} \pi(p, q)}{\partial^{2} q}\right|_{(p, \hat{q}(p))}$. However, the expression is complicated and it is hard to judge of its sign for an arbitrary $p$ from $\left[p_{l}, p_{h}\right]$. But important conclusions can be made when considering a limiting case with $p \rightarrow p_{m}$. Lemma A.4 tells that either $\left[p_{l}, p_{h}\right]=\left[p_{l}, p_{m}\right]$ or $\left[p_{l}, p_{h}\right]=\left[p_{m}, p_{h}\right]$. What is the case depends upon the sign of $U_{p q}\left(p_{m}, \hat{q}\left(p_{m}\right)\right)+\frac{1}{a} U_{q q}\left(p_{m}, \hat{q}\left(p_{m}\right)\right)$. This latter expression does not have an immediate interpretation but it can be rewritten as to allow for an economic one. Namely, this expression can be formulated in terms of a slope of a contract curve at $p_{m}$, which is $g^{\prime}\left(p_{m}\right)$. Lemma A.5 does so. Together with lemma A.4 they gives: if $g^{\prime}\left(p_{m}\right)<\frac{1}{a}$ then $\left[p_{l}, p_{h}\right]=\left[p_{l}, p_{m}\right]$ and if $g^{\prime}\left(p_{m}\right)>\frac{1}{a}$ then $\left[p_{l}, p_{h}\right]=\left[p_{m}, p_{h}\right]$. Finally, lemma A.2 gives that $U(p, \hat{q}(p))$ is strictly decreasing in $p$ for $p<p_{m}$ and is strictly increasing in $p$ for $p>p_{m}$.

Theorem 5. Consider an arbitrary strictly increasing, strictly convex and twice differentiable equilibrium curve $\hat{q}(p)$ defined over $\left[p_{l}, p_{h}\right]$ and satisfying $(38)$ or (39) or both. Then there exist such a utility function $U(p, q)$ satisfying assumption 1 , such parameters $\left(U_{R}, \lambda_{H}, \lambda_{M}, \lambda_{L}, a\right)$ and such off-the-equilibrium beliefs that there will be a corresponding exact signalling equilibrium, i.e one that has $\hat{q}(p)$ as its equilibrium curve. If (38) is satisfied, then $U(p, q),\left(U_{R}, \lambda_{H}, \lambda_{M}, \lambda_{L}, a\right)$ and offthe-equilibrium beliefs can be chosen such that lower prices signall higher utility in the equilibrium. If (39) is satisfied, they can be chosen such that higher prices signall higher utility in the equilibrium. If both conditions are satisfied, both types of an equilibrium can be achieved. 
Proof. We only discuss how to find such parameters of our model as to get an equilibrium where lower prices signal higher utility. Construction of an equilibrium where higher prices signal higher utility is analogous. To prove the theorem we have to find $\left(U_{R}, \lambda_{H}, \lambda_{M}, \lambda_{L}, a\right), U(p, q)$ and off-the-equilibrium beliefs such that a) $U(p, q)$ satisfies assumption $1, \mathrm{~b})$ the resulting equilibrium curve is precisely $\hat{q}(p)$ and the resulting boundary points are precisely $p_{l}$ and $p_{h}, \mathrm{c}$ ) the expected profits $\pi(p, q)$ attain their maximum over the equilibrium curve $\hat{q}(p)$. We proceed as follows. First, we choose some specific parameters $\left(U_{R}, \lambda_{H}, \lambda_{M}, \lambda_{L}, a\right)$ and we choose a specific utility function $U(p, q)$. Second, we show that a) and b) hold for those parameters and utility function. Third, we choose some specific but reasonable equilibrium beliefs and we show that c) holds as well. Take

$$
a=\frac{1}{\hat{q}^{\prime}\left(p_{h}\right)}
$$

Consider $\frac{d}{d p} \Pi(p, \hat{q}(p))$ :

$$
\frac{d}{d p} \Pi(p, \hat{q}(p))=1-a \hat{q}^{\prime}(p)=1-\frac{\hat{q}^{\prime}(p)}{\hat{q}^{\prime}\left(p_{h}\right)}
$$

We asked for $\hat{q}(p)$ to be strictly increasing and strictly convex, i.e. $\hat{q}^{\prime}(p)>0$ and $\hat{q}^{\prime \prime}(p)>0$. Therefore $\hat{q}^{\prime}(p)<\hat{q}^{\prime}\left(p_{h}\right)$ for $p<p_{h}$ and, consequently, $\frac{d}{d p} \Pi(p, \hat{q}(p))>0$ for $p<p_{h}$. In other words, equilibrium per-unit profits are strictly increasing in $p$ over $\left[p_{l}, p_{h}\right]$. Define

$$
\Pi_{l}=\Pi\left(p_{l}, \hat{q}\left(p_{l}\right)\right), \quad \Pi_{h}=\Pi\left(p_{h}, \hat{q}\left(p_{h}\right)\right)
$$

Take

$$
\lambda_{H}+\lambda_{M}=\frac{\Pi_{h}-\Pi_{l}}{\Pi_{h}+\Pi_{l}}, \quad \lambda_{L}=1-\left(\lambda_{H}+\lambda_{M}\right)=\frac{2 \Pi_{l}}{\Pi_{h}+\Pi_{l}}
$$

We choose precise values for $\lambda_{H}+\lambda_{M}$ and $\lambda_{L}$. As for $\lambda_{H}$ and $\lambda_{M}$, they can be chosen arbitrary but with $\lambda_{M}$ sufficiently small, more precisely, we take $\lambda_{M}$ such that

$$
\lambda_{M}<\left(\frac{\Pi_{l}}{\Pi_{h}}\right)^{2} \frac{\Pi_{h}-\Pi_{l}}{\Pi_{h}+\Pi_{l}}
$$

Trivially, $\lambda_{H}=\left(\lambda_{H}+\lambda_{M}\right)-\lambda_{M}$. Let

$$
D=\left\{(p, q): p \in\left[p_{l}, p_{h}\right], \Pi_{l} \leq \Pi(p, q) \leq \Pi_{h}\right\}
$$

To define $U(p, q)$ and to show that it satisfies assumption 1 we proceed as follows. First, we define $U(p, q)$ for $(p, q) \in D$ and we show that $U(p, q)$ satisfies assumption 1 on $D$. Second, we argue that $U(p, q)$ can be extended beyond $D$ in such a way that the assumption is still satisfied. As a result we will have a utility function $U(p, q)$ that satisfies assumption 1 in general and has an analytical expression for $(p, q) \in D$. Take

$$
U(p, q)=\frac{\Pi_{h}}{p-a q}-\frac{\lambda_{M}}{\lambda_{H}+\lambda_{M}} \frac{\Pi_{h}}{p-a \hat{q}(p)}-\frac{\lambda_{H}}{\lambda_{H}+\lambda_{M}} \quad \text { for } \quad(p, q) \in D
$$

and take $U_{R}=0$. Equilibrium curve $\hat{q}(p)$ was taken to be twice differentiable, hence $U(p, q)$ is also twice differentiable on $D$ as follows from its definition. Consider $U_{q}$ :

$$
U_{q}(p, q)=\frac{\Pi_{h}}{(p-a q)^{2}} \cdot a>0
$$


Hence, $U(p, q)$ is strictly increasing in $q$. Next, consider $U_{p}$ :

$$
U_{p}(p, q)=-\frac{\Pi_{h}}{(p-a q)^{2}}+\frac{\lambda_{M}}{\lambda_{M}+\lambda_{H}} \frac{\Pi_{h}}{(p-a \hat{q}(p))^{2}}\left(1-a \hat{q}^{\prime}(p)\right)
$$

For $(p, q) \in D$ it holds that

$$
p-a q \leq \Pi_{h}, \quad p-a \hat{q}(p) \geq \Pi_{l}, \quad 0 \leq \hat{q}^{\prime}(p) \leq \frac{1}{a}
$$

Therefore

$$
U_{p}(p, q) \leq-\frac{\Pi_{h}}{\Pi_{h}^{2}}+\frac{\lambda_{M}}{\lambda_{M}+\lambda_{H}} \frac{\Pi_{h}}{\Pi_{l}^{2}}<0,
$$

where the last inequality follows directly from (103) and (104). Hence, $U(p, q)$ is strictly decreasing in $p$ on $D$. If $U(p, q)$ is strictly decreasing in $p$ and strictly increasing in $q$ then it is strictly quasi-concave if and only if its iso-utility curves $\tilde{q}(p)$ are strictly convex, i.e. it should be that $\tilde{q}^{\prime \prime}(p)>0$. To check that $\tilde{q}^{\prime \prime}(p)>0$ we start with $\tilde{q}^{\prime}(p)$ :

$$
\tilde{q}^{\prime}(p)=-\frac{U_{p}(p, \tilde{q}(p))}{U_{q}(p, \tilde{q}(p))}=\frac{1}{a}-\frac{1}{a} \frac{\lambda_{M}}{\lambda_{M}+\lambda_{H}} \frac{(p-a \tilde{q}(p))^{2}}{(p-a \hat{q}(p))^{2}}\left(1-a \hat{q}^{\prime}(p)\right)
$$

Next,

$$
\begin{aligned}
& \tilde{q}^{\prime \prime}(p)= \\
& \qquad \begin{aligned}
& \frac{2}{a} \frac{\lambda_{M}}{\lambda_{M}+\lambda_{H}} \frac{(p-a \tilde{q}(p))^{2}\left(1-a \hat{q}^{\prime}(p)\right)^{2}}{(p-a \hat{q}(p))^{3}}(\left.1-\frac{\lambda_{M}}{\lambda_{M}+\lambda_{H}} \frac{p-a \tilde{q}(p)}{p-a \hat{q}(p)}\right)+ \\
& \frac{\lambda_{M}}{\lambda_{M}+\lambda_{H}} \frac{(p-a \tilde{q}(p))^{2}}{(p-a \hat{q}(p))^{2}} \hat{q}^{\prime \prime}(p)
\end{aligned}
\end{aligned}
$$

Equilibrium curve $\hat{q}(p)$ was taken to be strictly convex, so $\hat{q}^{\prime \prime}(p)>0$. Also, on $D$,

$$
p-a \tilde{q}(p) \leq \Pi_{h}, \quad p-a \hat{q}(p) \geq \Pi_{l}
$$

and then

$$
1-\frac{\lambda_{M}}{\lambda_{M}+\lambda_{H}} \frac{p-a \tilde{q}(p)}{p-a \hat{q}(p)} \geq 1-\frac{\lambda_{M}}{\lambda_{M}+\lambda_{H}} \frac{\Pi_{h}}{\Pi_{l}}>1-\frac{\Pi_{l}}{\Pi_{h}}>0,
$$

where the second inequality follows directly from (103) and (104). Consequently, $\tilde{q}^{\prime \prime}(p)>0$ and $U(p, q)$ is strictly quasi-concave. Consider $U(p, q)$ as a map of isoutility curves on $D$. These iso-utility curves, when viewed as functions of $p$, are strictly increasing, strictly convex and are as sufficiently smooth as to make $U(p, q)$ twice differentiable. Also, $D$ is a convex set. Clearly then, these iso-utility curves can be extended beyond $D$ as to still be strictly increasing, strictly convex and sufficiently smooth. Moreover, these iso-utility curves can be made convex enough outside $D$ so as to have each of them attain a slope of $\frac{1}{a}$ at some point. This latter condition guarantees that $\max _{(p, q)} \Pi(p, q)$ s.t. $U(p, q) \geq x$ has an inner solution. Summarising, there exists a utility function $U(p, q)$ satisfying assumption 1 and for $(p, q) \in D$ being explicitly defined by (106). We take such a $U(p, q)$ as our utility function. Now we proceed with verifying b). Given $\left(U_{R}, \lambda_{H}, \lambda_{M}, \lambda_{L}, a\right)$ and given $U(p, q)$ we can solve for the equilibrium curve and for the boundary points. 
We denote the equilibrium curve and the boundary points that we get as a solution to the model by $\hat{q}_{s}(p)$ and by $p_{l}^{s}, p_{h}^{s}$ respectively, this way we can distinguish them from the given $\hat{q}(p)$ and $p_{l}, p_{h}$. Then to verify b) means to verify that $\hat{q}_{s}(p) \equiv \hat{q}(p)$, $p_{l}^{s}=p_{l}$ and $p_{h}^{s}=p_{h}$. In general, $\left[p_{l}^{s}, p_{h}^{s}\right]=\left[p_{l}^{s}, p_{m}\right]$ in equilibria where lower prices signal higher utility - see theorem 3 . In our case we are also looking for such an equilibria, hence we also choose $p_{h}^{s}=p_{m} \cdot{ }^{15}$ Recollect that

$$
\left(p_{m}, q_{m}\right)=\arg \max _{p, q} \Pi(p, q) \quad \text { s.t. } \quad U(p, q) \geq U_{R}
$$

Utility $U(p, q)$ strictly increases in $q$ and strictly decreases in $p$, per-unit profits $\Pi(p, q)$ do just the opposite. Therefore, the solution is attained when $U(p, q)=U_{R}$ and the first-order conditions for this optimisation problem are:

$$
\left\{\begin{array}{l}
\frac{\Pi_{p}(p, q)}{\Pi_{q}(p, q)}=\frac{U_{p}(p, q)}{U_{q}(p, q)} \\
U(p, q)=U_{R}
\end{array}\right.
$$

Suppose optimal $(p, q) \in D$. Then using (106) and simplifying gives:

$$
\left\{\begin{array}{l}
1-a \hat{q}^{\prime}(p)=0, \\
\frac{\Pi_{h}}{p-a q}-\frac{\lambda_{M}}{\lambda_{H}+\lambda_{M}} \frac{\Pi_{h}}{p-a \hat{q}(p)}-\frac{\lambda_{H}}{\lambda_{H}+\lambda_{M}}=0 .
\end{array}\right.
$$

Given that $a=\frac{1}{\hat{q}^{\prime}\left(p_{h}\right)}$ and that $\Pi_{h}=p_{h}-a \hat{q}\left(p_{h}\right)$ it is straightforward to verify that point $\left(p_{h}, \hat{q}\left(p_{h}\right)\right)$ satisfies (117). Moreover, from assumption 1 it follows that there is a unique solution to the considered optimisation problem, therefore $\left(p_{h}, \hat{q}\left(p_{h}\right)\right)$ is this unique solution. So, $\left(p_{m}, q_{m}\right)=\left(p_{h}, \hat{q}\left(p_{h}\right)\right)$ and $p_{h}^{s}=p_{h}$. From theorem 2

$$
\hat{q}_{s}^{\prime}(p)=-\frac{\lambda_{H}+\lambda_{M}}{\lambda_{M}} \frac{U_{p}\left(p, \hat{q}_{s}(p)\right)}{U_{q}\left(p, \hat{q}_{s}(p)\right)}-\frac{1}{a} \frac{\lambda_{H}}{\lambda_{M}}
$$

The boundary condition comes from lemma A.1: $\hat{q}_{s}(p)$ has to go through the point $\left(p_{m}, q_{m}\right)=\left(p_{h}, \hat{q}\left(p_{h}\right)\right)$. For $p \in\left[p_{l}, p_{h}\right]$ we use (108) and (107) to rewrite (118) as

$$
\hat{q}_{s}^{\prime}(p)=\frac{1}{a}-\frac{1}{a}\left(\frac{p-a \hat{q}_{s}(p)}{p-a \hat{q}(p)}\right)\left(1-a \hat{q}^{\prime}(p)\right)
$$

Clearly, for $p \in\left[p_{l}, p_{h}\right] \hat{q}_{s}(p) \equiv \hat{q}(p)$ is a solution. Moreover, it is unique by the Picard's theorem. We can not say what $\hat{q}_{s}(p)$ is for $p \notin\left[p_{l}, p_{h}\right]$, but as we will see later on this is not required. The lower bound $p_{l}^{s}$ is implicitly defined by $U\left(p_{l}^{s}, \hat{q}_{s}\left(p_{l}^{s}\right)\right)=U_{h}$ and $U_{h}$ comes from $F\left(U_{h}\right)=1$. We now solve these equations. For $p \in\left[p_{l}, p_{h}\right]$

$$
U\left(p, \hat{q}_{s}(p)\right)=U(p, \hat{q}(p))=\frac{\lambda_{H}}{\lambda_{H}+\lambda_{M}}\left(\frac{\Pi_{h}}{p-a \hat{q}(p)}-1\right)
$$

\footnotetext{
${ }^{15}$ This is a subtle point. In our case utility function $U(p, q)$, as defined in (106), violates assumption 3, therefore theorem 3 can not be applied. Consequently, there is no immediate restriction on the choice of $p_{h}^{s}$ : we are free to choose $p_{h}^{s}=p_{m}$, but other choices are also possible. Such a utility function is a special case, however. It is possible to perturb the suggested $U(p, q)$ a bit in such a way that the current theorem still holds, assumption 3 is not violated and theorem 3 dictates a unique choice of $p_{h}^{s}$, namely $p_{h}^{s}=p_{m}$. See the following discussion on profits and footnote 17 for a brief explanation on how to do it.
} 
From theorem 1

$$
F(u)=\frac{1}{2} \frac{\lambda_{L}}{\lambda_{H}+\lambda_{M}}\left(\frac{\Pi_{h}}{\hat{\Pi}(u)}-1\right)
$$

where

$$
\hat{\Pi}(u)=\tilde{p}(u)-a \hat{q}(\tilde{p}(u))
$$

and $\tilde{p}(u)$ is implicitly defined by

$$
U(\tilde{p}(u), \hat{q}(\tilde{p}(u)))=u .
$$

Substituting $p$ with $\tilde{p}(u)$ in (120) and the resulting $\tilde{p}(u)-a \hat{q}(\tilde{p}(u))$ with $\hat{\Pi}(u)$ and then comparing the outcome with (121) gives

$$
F(u)=\frac{1}{2} \frac{\lambda_{L}}{\lambda_{H}} \cdot u
$$

Hence $U_{h}=2 \frac{\lambda_{H}}{\lambda_{L}}$. Suppose $p_{l}^{s} \in\left[p_{l}, p_{h}\right]$, then using (103) and (104) we can rewrite $U\left(p_{l}^{s}, \hat{q}\left(p_{l}^{s}\right)\right)=U_{h}$ as

$$
p_{l}^{s}-a \hat{q}\left(p_{l}^{s}\right)=\Pi_{l}=p_{l}-a \hat{q}\left(p_{l}\right)
$$

Clearly then, $p_{l}^{s}=p_{l}$ is a solution. Moreover, it is unique because $U(p, \hat{q}(p))$ is strictly monotone in $p$ (lemma A.2). Next, we have to verify c), i.e. we have to verify that the expected profits $\pi(p, q)$ attain their maximum over $\hat{q}(p)$. When the opponent is playing the equilibrium strategy

$$
\pi(p, q)=\left(F(U(p, q)) \cdot \lambda_{H}+F(\hat{U}(p)) \cdot \lambda_{M}+\frac{\lambda_{L}}{2}\right) \Pi(p, q),
$$

where $\hat{U}(p)$ stands for the utility that partially informed consumers expect to receive given that the price is $p$. For $p \in\left[p_{l}, p_{h}\right]$ we have that

$$
\hat{U}(p)=U\left(p, \hat{q}_{s}(p)\right)=U(p, \hat{q}(p)) .
$$

Since $U(p, \hat{q}(p))$ is strictly decreasing in $p$ for $p \in\left[p_{l}, p_{h}\right]$ we can choose and we choose such off-the-equilibrium beliefs that $\hat{U}(p)$ is decreasing in $p$ for $p \in \mathbb{R}^{16}$ Define

$$
\begin{aligned}
& S_{C}=\left\{(p, q): p \in\left[p_{l}, p_{h}\right], U_{R} \leq U(p, q) \leq U_{h}\right\}, \\
& S_{B}=\left\{(p, q): U(p, q)<U_{R}\right\}, \\
& S_{L}=\left\{(p, q): p<p_{l}, U_{R} \leq U(p, q) \leq U_{h}\right\}, \\
& S_{R}=\left\{(p, q): p>p_{h}, U_{R} \leq U(p, q) \leq U_{h}\right\}, \\
& S_{T}=\left\{(p, q): U(p, q)>U_{h}\right\} .
\end{aligned}
$$

Clearly, $\bigcup_{x} S_{x}=\mathbb{R}^{2}$. We consider $\pi(p, q)$ over each of these regions in turn. First, suppose $(p, q) \in S_{C}$. For $p \in\left[p_{l}, p_{h}\right]$ it holds that $\Pi_{l} \leq p-a \hat{q}(p) \leq \Pi_{h}$. Then, using the definitions for $\lambda_{H}+\lambda_{M}$ and $\lambda_{L}$, it is straightforward to verify that

$$
\begin{aligned}
& U(p, q) \geq U_{R} \Rightarrow \Pi(p, q) \leq \Pi_{h}, \\
& U(p, q) \leq U_{h} \Rightarrow \Pi(p, q) \geq \Pi_{l} .
\end{aligned}
$$

\footnotetext{
${ }^{16}$ These are reasonable off-the-equilibrium beliefs as they depend upon $p$ in the same direction as on-the-equilibrium beliefs do.
} 
Consequently, $S_{C} \subseteq D$. But for $(p, q) \in D$ we have an explicit expression for $U(p, q)$ and $\hat{U}(p)=U(p, \hat{q}(p))$ for $p \in\left[p_{l}, p_{h}\right]$. Also, $F(u)=\frac{1}{2} \frac{\lambda_{L}}{\lambda_{H}} \cdot u$ for $U_{R} \leq u \leq U_{h}$. Expanding (126) then gives:

$$
\begin{gathered}
\pi(p, q)=\left(F(U(p, q)) \cdot \lambda_{H}+F(U(p, \hat{q}(p))) \cdot \lambda_{M}+\frac{\lambda_{L}}{2}\right) \cdot(p-a q)= \\
\frac{\lambda_{L}}{2}\left(U(p, q)+\frac{\lambda_{M}}{\lambda_{H}} U(p, \hat{q}(p))+1\right) \cdot(p-a q)=\frac{\lambda_{L}}{2} \Pi_{h},
\end{gathered}
$$

where the last equality follows directly from the definitions of $U(p, q), \lambda_{H}+\lambda_{M}$, $\lambda_{L}$ and $\Pi_{h}, \Pi_{l}$, see equations (106), (103) and (102). So, the expected profits are constant for $(p, q) \in S_{C}$. Next, suppose $(p, q) \in S_{B}$. But then $U(p, q)<U_{R}$, so no consumers buy the product and

$$
\pi(p, q)=0<\frac{\lambda_{L}}{2} \Pi_{h}
$$

Next, suppose $(p, q) \in S_{L}$. This implies $U_{R} \leq U(p, q) \leq U_{h}$. Let

$$
\begin{aligned}
q_{u} & =\hat{q}\left(p_{l}\right), \\
q_{b} & =\frac{1}{a}\left(p_{l}-\frac{\left(\lambda_{H}+\lambda_{M}\right) \Pi_{l} \Pi_{h}}{\lambda_{M} \Pi_{h}+\lambda_{H} \Pi_{l}}\right) .
\end{aligned}
$$

Then it directly follows from the definitions of $U(p, q), U_{R}, \lambda_{H}+\lambda_{M}, \lambda_{L}$ and $\Pi_{h}$, $\Pi_{l}$ that $U\left(p_{l}, q_{u}\right)=U_{h}$ and $U\left(p_{l}, q_{b}\right)=U_{R}$. Moreover, $U\left(p_{l}, q\right)$ is continuous in $q$ and therefore there exists $q^{*}$ such that

$$
U\left(p_{l}, q^{*}\right)=U(p, q)
$$

Consequently,

$$
F(U(p, q))=F\left(U\left(p_{l}, q^{*}\right)\right) .
$$

Since $p<p_{l}$ and since $\hat{U}(p)$ is decreasing in $p, \hat{U}(p) \geq \hat{U}\left(p_{l}\right)$. But $\hat{U}\left(p_{l}\right)=$ $U\left(p_{l}, \hat{q}\left(p_{l}\right)\right)=U_{h}$ and $F(u)=1$ for $u \geq U_{h}$. So,

$$
F(\hat{U}(p))=F\left(\hat{U}\left(p_{l}\right)\right) .
$$

Given (139), we can take an iso-utility curve $\tilde{q}(p)$ going through points $(p, q)$ and $\left(p_{l}, q^{*}\right)$. From (111) we have that

$$
\tilde{q}^{\prime}\left(p_{l}\right)=\frac{1}{a}-\frac{1}{a} \frac{\lambda_{M}}{\lambda_{M}+\lambda_{H}} \frac{\left(p_{l}-a \tilde{q}\left(p_{l}\right)\right)^{2}}{\Pi_{l}^{2}}\left(1-a \hat{q}^{\prime}\left(p_{l}\right)\right) .
$$

As $\hat{q}^{\prime}\left(p_{l}\right)<\frac{1}{a}$ it follows that $\tilde{q}^{\prime}\left(p_{l}\right)<\frac{1}{a}$. Utility $U(p, q)$ satisfies assumption 1 and therefore $\tilde{q}^{\prime \prime}(p)>0$. So, $\tilde{q}^{\prime}(p)<\frac{1}{a}$ for all $p \leq p_{l}$. Consequently,

$$
\frac{d}{d p} \Pi(p, \tilde{q}(p))=1-a \tilde{q}^{\prime}(p)>0 \quad \text { for all } \quad p \leq p_{l} .
$$

As $p<p_{l}$ we then have that

$$
\Pi(p, q)<\Pi\left(p_{l}, q^{*}\right) .
$$


Bringing together (140), (141) and (144) and noticing that $\left(p_{l}, q^{*}\right) \in S_{C}$ gives:

$$
\begin{aligned}
\pi(p, q)= & \left(F(U(p, q)) \cdot \lambda_{H}+F(\hat{U}(p)) \cdot \lambda_{M}+\frac{\lambda_{L}}{2}\right) \Pi(p, q)< \\
& \left(F\left(U\left(p_{l}, q^{*}\right)\right) \cdot \lambda_{H}+F\left(\hat{U}\left(p_{l}\right)\right) \cdot \lambda_{M}+\frac{\lambda_{L}}{2}\right) \Pi\left(p_{l}, q^{*}\right)=\frac{\lambda_{L}}{2} \Pi_{h} .
\end{aligned}
$$

Next, suppose $(p, q) \in S_{R}$. This case is analogous to the previous one and we also get that

$$
\pi(p, q)<\frac{\lambda_{L}}{2} \Pi_{h}
$$

Finally, suppose $(p, q) \in S_{T}$, i.e. $U(p, q)>U_{h}$. Given that $U(p, q)$ satisfies assumption 1 there exists $q^{*}<q$ such that $U\left(p, q^{*}\right)=U_{h}$. As $F(u)=1$ for $u \geq U_{h}$ we have that $F(U(p, q))=F\left(U\left(p, q^{*}\right)\right)$. Trivially, $\Pi(p, q)<\Pi\left(p, q^{*}\right)$. Therefore,

$$
\begin{aligned}
\pi(p, q)= & \left(F(U(p, q)) \cdot \lambda_{H}+F(\hat{U}(p)) \cdot \lambda_{M}+\frac{\lambda_{L}}{2}\right) \Pi(p, q)< \\
& \left(F\left(U\left(p, q^{*}\right)\right) \cdot \lambda_{H}+F(\hat{U}(p)) \cdot \lambda_{M}+\frac{\lambda_{L}}{2}\right) \Pi\left(p, q^{*}\right)=\pi\left(p, q^{*}\right) .
\end{aligned}
$$

But $\left(p, q^{*}\right) \in S_{L} \cup S_{C} \cup S_{R}$, so $\pi\left(p, q^{*}\right) \leq \frac{\lambda_{L}}{2} \Pi_{h}$ and $\pi(p, q)<\frac{\lambda_{L}}{2} \Pi_{h}$. Given that $\pi(p, q)=\frac{\lambda_{L}}{2} \Pi_{h}$ for $(p, q) \in S_{C}$, that $\pi(p, q)<\frac{\lambda_{L}}{2} \Pi_{h}$ for $(p, q) \notin S_{C}$ and that the equilibrium curve $\hat{q}(p)$ belongs to $S_{C}$, we have that the profits attain their maximum over $\hat{q}(p)$, though not exclusively. ${ }^{17}$

\footnotetext{
${ }^{17}$ It is possible to construct such a utility function that $\pi(p, q)$ will attain its unique maximum over $\hat{q}(p)$. Roughly speaking, one has to begin with $U(p, q)$ and then make its iso-utility curves more convex around the equilibrium curve $\hat{q}(p)$. Obviously, a) will still hold. When iso-utility curves are bend around $\hat{q}(p)$, they will coincide at $\hat{q}(p)$ with the original iso-utility curves of $U(p, q)$ in their first order approximation, so b) will still hold. Also, each point from the original iso-utility curve can be viewed as going into the point on a new iso-utility curve with the same $p$, same $U(p, q)$ but with strictly higher $q$ (except for the points of $\hat{q}(p))$, so profits $\pi(p, q)$ will decrease around the equilibrium curve.
} 